\title{
Route Identification Method for On-Ramp Traffic at Adjacent Intersections of Expressway Entrance
}

\author{
Wenxuan Wang $\mathbb{D}^{1},{ }^{1}$ Xiaodong Zhu $\mathbb{D}^{2},{ }^{2}$ Yanli Wang $\mathbb{D}^{1},{ }^{1}$ and Bing Wu $\mathbb{D}^{1}$ \\ ${ }^{1}$ Key Laboratory of Road and Traffic Engineering of Ministry of Education, Tongji University, Shanghai 201804, China \\ ${ }^{2}$ China Highway Engineering Consultants Corporation, Beijing 100089, China \\ Correspondence should be addressed to Yanli Wang; wangyanli@tongji.edu.cn and Bing Wu; wubing@tongji.edu.cn
}

Received 12 January 2019; Revised 17 April 2019; Accepted 10 August 2019; Published 4 December 2019

Academic Editor: Monica Menendez

Copyright ( 2019 Wenxuan Wang et al. This is an open access article distributed under the Creative Commons Attribution License, which permits unrestricted use, distribution, and reproduction in any medium, provided the original work is properly cited.

\begin{abstract}
To determine the control strategy at intersections adjacent to the expressway on-ramp, a route identification method based on empirical mode decomposition (EMD) and dynamic time warping (DTW) is established. First, the de-noise function of EMD method is applied to eliminate disturbances and extract features and trends of traffic data. Then, DTW is used to measure the similarity of traffic volume time series between intersection approaches and expressway on-ramp. Next, a three-dimensional feature vector is built for every intersection approach traffic flow, including DTW distance, space distance between on-ramp and intersection approach, and intersection traffic volume. Fuzzy C-means clustering method is employed to cluster intersection approaches into classifications and identify critical routes carrying the most traffic to the on-ramp. The traffic data are collected by inductive loops at Xujiahui on-ramp of North and South Viaduct Expressway and surrounding intersections in Shanghai, China. The result shows that the proposed method can achieve route classification among intersections for different time periods in one day, and the clustering result is significantly influenced by three dimensions of traffic flow feature vector. As an illustrative example, micro-simulation models are built with different control strategies. The simulation shows that the coordinated control of critical routes identified by the proposed method has a better performance than coordinated control of arterial roads. Conclusions demonstrated that the proposed route identification method could provide a theoretical basis for the coordinated control of traffic signals among intersections and on-ramp.
\end{abstract}

\section{Introduction}

With increasing congestion problems of urban traffic, many expressway on-ramp are usually congested, especially during the morning and evening peak periods. Congestion is frequently spread to ground intersections or roads through the oversaturated on-ramp. To solve this problem, scholars began to pay attention to coordinated control of on-ramp and adjacent intersections to improve the overall operation efficiency of traffic system [1]. However, most current studies only coordinately control the on-ramp and one connected intersection [2]. To achieve coordinated control of an on-ramp and intersections in an adjacent area, we can only refer to the research methods and findings of the coordinated control among intersections.

Two coordinated control modes of intersections including subarea control and arterial road control are widely used [3]. In subarea control mode, a large area is divided into small areas, and internal function or state within these subareas are similar. This control mode can achieve real-time traffic control and improve the stability of the system. The sub-area division process firstly chooses a key intersection on the basis of traffic volume, then the division will be finished based on principles of distance, saturation, and the correlation of key intersection and intersections [4]. The subarea division is to identify whether control performance can be improved when subarea coordinated mode is implemented to adjacent intersections. If the performance can be improved, the adjacent intersections should be divided into one subarea. Therefore, a straightforward method for subarea division is to calculate and compare different control performances of different signal-controlling modes. This method is time-consuming for a large area of intersections. Subarea control method can improve the efficiency of traffic in an intersection area [5]. But for the directional traffic flow from intersections to on-ramp, it involves not only the intersection area, but also the on-ramp connecting expressway and ground system. The sub-area control can not solve this problem effectively. The second coordinated control mode is arterial road control, it can achieve the control of 
intersections on arterial roads easily with green wave. But this method only simply considers the line control on arterial roads, and it cannot guarantee the best effect in an area when some branch roads with high traffic demand also need to be controlled.

By comparison of the above two control modes, neither of them is suitable for the coordinated control of on-ramp and adjacent intersections. Therefore, an effective way to coordinately control the traffic from intersections to on-ramp is necessary. Here, a critical routes identification and control method is proposed for on-ramp traffic at adjacent intersections. For on-ramp, critical routes [6] mean the routes carrying the maximal flow to on-ramp, and they are the key to determine the efficiency of the traffic flow to on-ramp. With route identification method, critical routes are regarded as the control objects with best effects. By controlling these critical routes, the most direct control effect among on-ramp and adjacent intersections can be achieved. We can use different control strategies based on different traffic demands. When the on-ramp is not congested, traffic signals on critical routes can be controlled to facilitate more vehicles flow to on-ramp. When vehicles are queuing on the ramp, the critical routes can be controlled to delay vehicle flow to on-ramp. Critical routes may not consistent with the direction of arterial roads, and they are not fixed. Critical routes may constantly vary with time, for example, they are different during peak hour and off-peak hour. Therefore, the fast and accurate identification of critical routes is an effective way to optimize the performance of traffic signals coordinated control.

For coordinated control of traffic signals at intersections, the method of critical routes identification should be dynamically and easily operated for various traffic states. And the method should be less affected by external environment. Commonly used route identification methods include traffic survey statistical analysis [3], real-time traffic surveillance method [7], floating car method [8], traffic count OD estimation method [9], vehicle plate automatic recognition technology [10] and the meta-heuristic search mechanism [11]. Although the above methods can identify critical routes at intersections, they are easily interfered by external environment or artificial factors. So, the above methods cannot meet the requirements of signal coordination control.

In this paper, a novel method is presented to identify the critical routes carrying the maximal traffic flow to on-ramp. And through signal coordinated control, the traffic efficiency among on-ramp and adjacent intersections can be improved. The new method utilizes dynamic time warping (DTW) distance to compare the similarity between on-ramp and adjacent intersection approaches. DTW is a method widely used in other areas such as speech recognition [12] and on-line signature recognition [13]. It is a classical and well-established distance measurement between two time series. Compared with classic Euclidean distance, DTW distance can measure similarity between two discrete time series which may have different time interval. A detailed description about DTW is provided in Section 3. The empirical mode decomposition (EMD) method is used to denoise the original time series. EMD can reconstruct the original traffic data and extract features and trends of them. Previous researchers have proposed

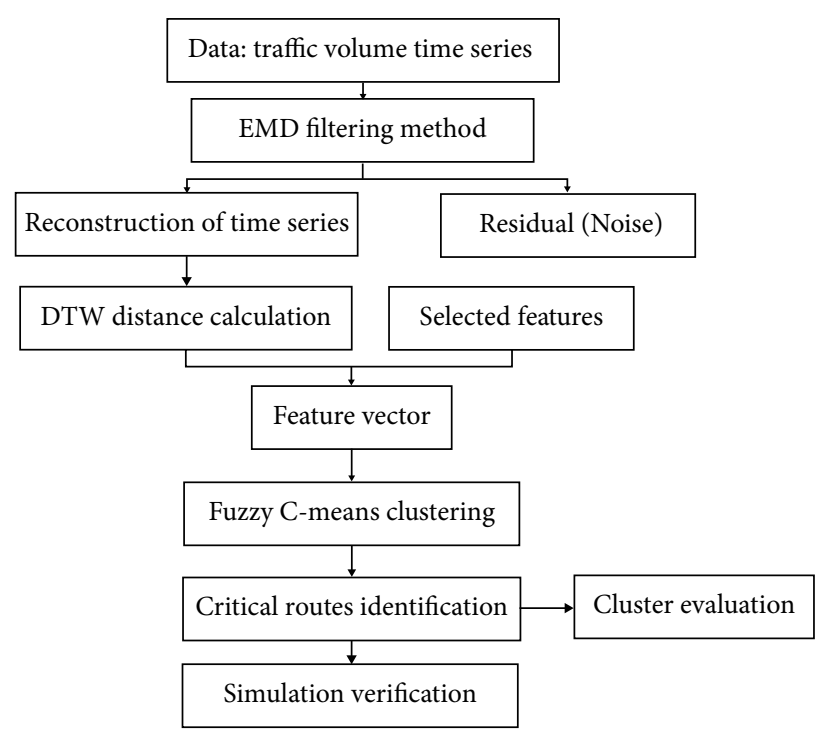

FIgURE 1: Architecture of this paper.

some filtering methods, such as linear method which is easy to design and implement but not very effective [14]. To overcome this shortcoming, nonlinear methods including wavelet filter are proposed, but the wavelet has the fixed basic function, so it cannot match all real data time series [15]. Recently, the data-driven EMD method was introduced from nonstationary and nonlinear process. The basic functions of EMD are derived from the data time series itself, and it's the main advantage of EMD. Detailed information about EMD is also introduced in Section 3.

The main framework of the paper is organized as follows. In the next section, a literature review on the coordinated control between expressway on-ramp and intersections is given. After that, three methods including EMD method, DTW distance method and fuzzy C-means clustering method are introduced. Then, data source is descripted for case study. The three methods are employed for a case study and the case results analysis is given in Section 5. Finally, the method is validated by traffic simulation in Section 6, and some conclusions are discussed in the last section. The architecture of this paper is shown as Figure 1.

\section{Literature Review}

Most of the research about coordinated control of on-ramp and adjacent intersections only focus on the closest intersection connected to the on-ramp $[16,17]$. The coordinated control includes ramp control [16], coordinated control of ramp and one connected intersection [2], coordinated control of ramp, expressway main line and one connected ground intersection [17]. In fact, when the vehicles queue on the ramp spreads to ground roads, the congestion will affect the traffic state of the one connected and further intersections. Few studies have focused on the coordinated control of on-ramp and intersections in adjacent areas.

If we want to achieve coordinated control of on-ramp and adjacent intersections, the research of coordinated control 
among intersections can be taken as a reference. One important method to coordinately control intersections is to measure correlation degree between intersections and then decide control priority. Current research mainly includes two methods to measure correlation degree between intersections. The first one is to build correlation degree models based on various influencing factors, including traffic volume, section length [16], section flow and cycle ratio [17]. The correlation degree model is very helpful to quantify the correlation degree between intersections, and more and more factors are needed to take into consideration to achieve a more accurate result. The other one is a data-driven method to measure the correlation degree between intersections by measuring similarity degree of traffic feature time series. Similarity degree is usually described by distance between two time series, and the most commonly used distances include Euclidean distance, DTW distance and editing distance [18]. For the traffic feature time series at different intersection, there will be asynchronism because of the lag between upstream and downstream traffic flow. Considering this, DTW distance is the best choice to analyse the similarity degree of traffic feature. Tin et al. [19] calculates DTW distance of different vehicles' speed time series to detect abnormal running speed of vehicles in advance. Zhang et al. [20] adopts the DTW distance to measure the similarity, and proposes a speed-up version of DTW to improve the accuracy of speed estimation and reduce the runtime.

After comparing two methods, it can be shown that the first correlation degree model method can measure the correlation degree between intersections by considering different influencing factors. This method is suitable for arterial intersections or a few connected intersections. However, for the correlation degree between the on-ramp and a large area of intersections, this method cannot behave well because of too many influencing factors and complex computation. It is hard to consider all the external influencing factors. By contrast, the similarity degree of traffic feature time series method is more appropriate to measure correlation degree between on-ramp and intersections. Even a large-scale area study will not add much complexity and the algorithm will not change with the external influencing factors.

On the basis of similarity degree, routes among intersections can be divided into different classifications. In previous studies, Lu et al. used a new data-driven method to measure road traffic correlation degree considering spatial heterogeneity [21] to achieve the dynamic division method for signal coordination. Yan et al. used spectrum analysis [22] which can determine the relevance and lag between upstream and downstream traffic to determine the critical routes among intersections. In addition, clustering is also a useful method to identify critical routes. Fuzzy-C means clustering method has been used to improve accuracy and robustness of sub-area control [23]. Clustering method is easy to calculate, and the number of clusters can be adjusted based on actual management requirements. So, clustering method can be used to classify different control levels and provide a reference for traffic management and control.

Therefore, in this paper, the similarity degree measurement based on DTW and fuzzy-C means clustering method can be integrated to achieve the route identification.

\section{Materials and Methods}

The principal methods used in this paper are the following: (1) EMD method that can filter and reconstruct traffic volume time series; (2) DTW distance that can measure similarity between traffic volume time series of intersection approaches and on-ramp; (3) fuzzy C-means method that can cluster intersection approaches into three classifications to identify critical routes.

\subsection{Empirical Mode Decomposition (EMD)}

3.1.1. Time Series. A time series is a sequence of real data observed in a period of time. These values are recorded with intervals between them, such as a time series $T_{n}=\left\{t_{1}, t_{2} \ldots, t_{n}\right\}$. In this work, a sequence of traffic volumes is considered as a time series.

3.1.2. Introduction of EMD. Empirical mode decomposition (EMD) [24] is a data analysis method with which any complicated data set can be decomposed into a small number of intrinsic mode functions (IMF) through sifting process. Each IMF has a certain time scale distinct from others [25]. EMD picks out the highest frequency oscillation from the time series as the first IMF and continue to pick out the highest frequency part in the remaining part of data to be the second IMF, and so on. It is apparent that IMFs's frequencies show a decrease trend step by step. This decomposition method is adaptive and highly efficient. A function needs to satisfy two conditions to be an IMF, readers can refer to [24].

EMD process involves five major steps as following.

Step 1. Identify all the local extrema of original time series $x(t)$, then obtain the upper envelope $u_{1}(t)$ and the lower envelope $l_{1}(t)$.

Step 2. The point-by-point envelope mean $m_{1}(t)$ can be calculated using the upper and lower envelopes as follows:

$$
m_{1}(t)=\frac{u_{1}(t)+l_{1}(t)}{2} .
$$

Step 3. Using the original time series $x(t)$ and $m_{1}(t)$ to get $h_{1}(t)$ as follows:

$$
h_{1}(t)=x(t)-m_{1}(t)
$$

Step 4. If $h_{1}(t)$ satisfies the definition of IMF, $h_{1}(t)$ can be regarded as the first IMF $c_{1}(t)$ and then residue $r_{1}(t)$ is substituted for the original $x(t)$, where

$$
r_{1}(t)=x(t)-c_{1}(t)
$$

If $h_{1}(t)$ is not an IMF, $h_{1}(t)$ should be seen as the original time series and Steps 1-3 are repeated.

Step 5. Repeat Steps 1-4 and stop when the residue $r_{n}(t)$ becomes a monotonic function.

Step 6. The original time series $x(t)$ can be decomposed into $n$ modes and a residue as follows:

$$
x(t)=\sum_{k=1}^{n} c_{k}(t)+r_{n}(t) .
$$




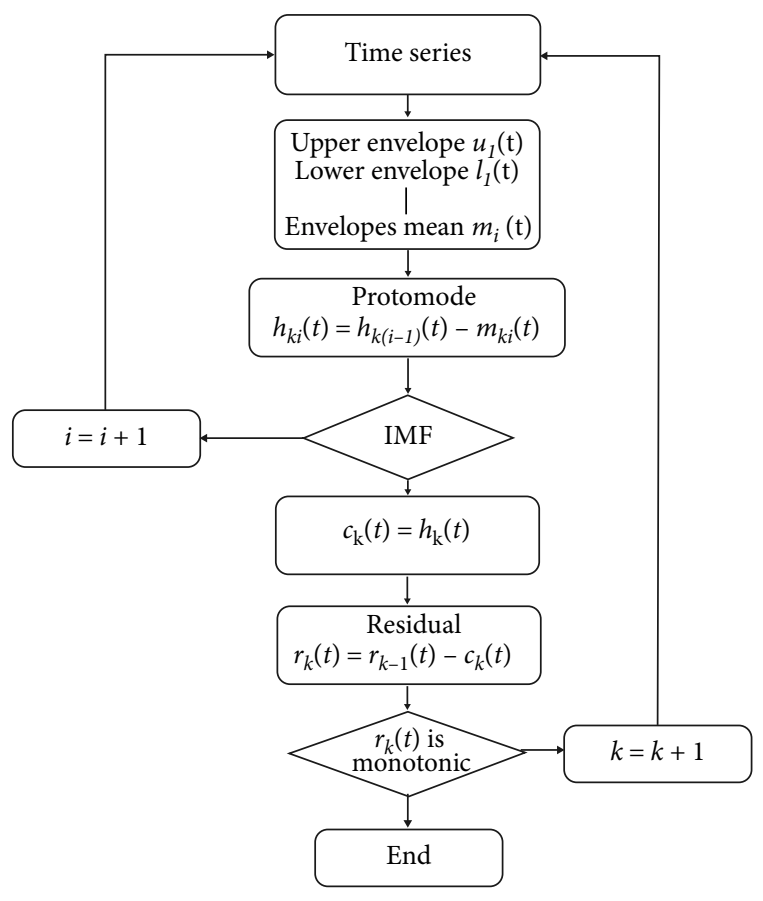

FIGURE 2: Flowchart of EMD.

Thus, the original data are decomposed into $n$ IMFs with different frequencies ranging from high to low and a residue $r_{n}(t)$ obtained with mean trend of original data.

The detail flowchart is listed in Figure 2. Take traffic volume time series as an example (see Figure 3), it is decomposed by EMD into eight IMFs and one residue. One can observe that the IMF construction starts from the highest frequency component, and the last extracted function is monotone.

3.1.3. Filtering Method. Boudraa and Cexus [25] introduced an EMD-based method to de-noise and smooth the data set. Being fully data-driven, this method does not need to set any threshold or select wavelet basis function. Thus, EMD is much more efficient than wavelet filtering method. Given a set of data $x(t)$ containing the deterministic part and white noise, we need to extract the deterministic part from $x(t) . x(t)$ can be decomposed into $n$ IMFs and a residue $r_{n}(t)$ by EMD, and we can get the reconstruction $\tilde{x}(t)$ with the $k$-th $\sim n$-th IMFs and $r_{n}(t)$ (Equation (5)).

$$
\tilde{x}_{k}(t)=\sum_{i=k}^{n} \operatorname{IMF}_{i}(t)+r_{n}(t), \quad k=2, \ldots, n .
$$

To confirm the value of $k$, consecutive mean square error (CMSE) [24] is proposed to filter data by measuring the squared Euclidean distance between two consecutive reconstructions of the data set. CMSE is defined as follows:

$$
\operatorname{CMSE}\left(\tilde{x}_{k}, \tilde{x}_{k+1}\right)=\frac{1}{T} \sum_{t=1}^{T}\left[\tilde{x}_{k}(t)-\tilde{x}_{k+1}(t)\right]^{2}=\frac{1}{T} \sum_{t=1}^{T} \operatorname{IMF}_{k}^{2}(t)
$$

where $k=1, \ldots, n-1, T$ is the length of $x(t)$. If

$$
j=\underset{1<k<n-1}{\operatorname{argmin}}\left[\operatorname{CMSE}\left(\tilde{x}_{k}, \tilde{x}_{k+1}\right)\right] .
$$

Then we estimate $\tilde{x}(t)$ as:

$$
\tilde{x}(t)=\tilde{x}_{j}(t)=\sum_{i=j}^{n} \operatorname{IMF}_{i}(t)+r_{n}(t) .
$$

\subsection{Dynamic Time Warping (DTW)}

3.2.1. Distance Measures of Time Series. Distance measure has been applied in similarity search, classification, and clustering of time series. There have been many distance measurements and improved methods to measure the similarity between time series. Among these distance measures, Euclidean distance and DTW distance are widely used because they were proved hard to be beaten [26, 27]. For Euclidean distance, the similarity between two time series $T=\left\{t_{1}, t_{2} \ldots, t_{n}\right\}$ and $S=\left\{s_{1}, s_{2} \ldots, s_{n}\right\}$ is defined as follows:

$$
E D \_d i s(T, S)=\sqrt{\sum_{i=1}^{n}\left(t_{i}-s_{i}\right)^{2}} \text {. }
$$

Note that two time series $T$ and $S$ must have the same length of $n$.

Euclidean distance is unsuitable for time series distance measure in case time series data sets are of different lengths or they are distorted along the time axis. For traffic flow, there must be time lag between upstream and downstream time series, and two time series are not corresponding to each other point to point on time axis. Therefore, we use DTW distance to avoid these problems.

3.2.2. Introduction of DTW Distance. DTW distance is a measure that allows non-point-to-point alignment between two time series to accommodate sequences that are similar but out of phase [28].

Given two traffic volume time series $T(m)$ and $S(n): T(m)=\left\{t_{1}, t_{2}, \ldots t_{i}, \ldots, t_{m}\right\}, S(n)=\left\{s_{1}, s_{2}, \ldots s_{i}, \ldots, s_{n}\right\}$. DTW is a dynamic programming technique which attempts to find all possible warping paths between two time series to get a minimum distance. The difference between DTW and Euclidean distance is shown in Figure 4. The shape of two time series are similar but not aligned in time axis, and DTW distance $a-b$ is more intuitive than Euclidean distance $a-b^{\prime}$, a pessimistic dissimilarity measure (see Figure 4 ).

Now we would like to show how to compute DTW distance between two time series. First of all, we construct a cumulative warping distance matrix $D$ with size $m \times n$. Then, every element in matrix $D$ is computed as follows:

$$
\left\{\begin{array}{c}
D(1,1)=d(1,1), \\
D(i, 1)=d(i, 1)+D(i-1,1), \quad \text { if } i<1, \\
D(1, j)=d(1, j)+D(1, j-1), \quad \text { if } j<1, \\
D(i, j)=d(i, j)+\min [D(i-1, j), D(i, j-1), \quad D(i-1, j-1)],
\end{array}\right.
$$

where $D(i, j)$ means the iterative computation of the minimum cumulative distance of two time series from starting point $(1,1)$ to point $(i, j)$. And $d(i, j)$ is the distance between the $i$-th 


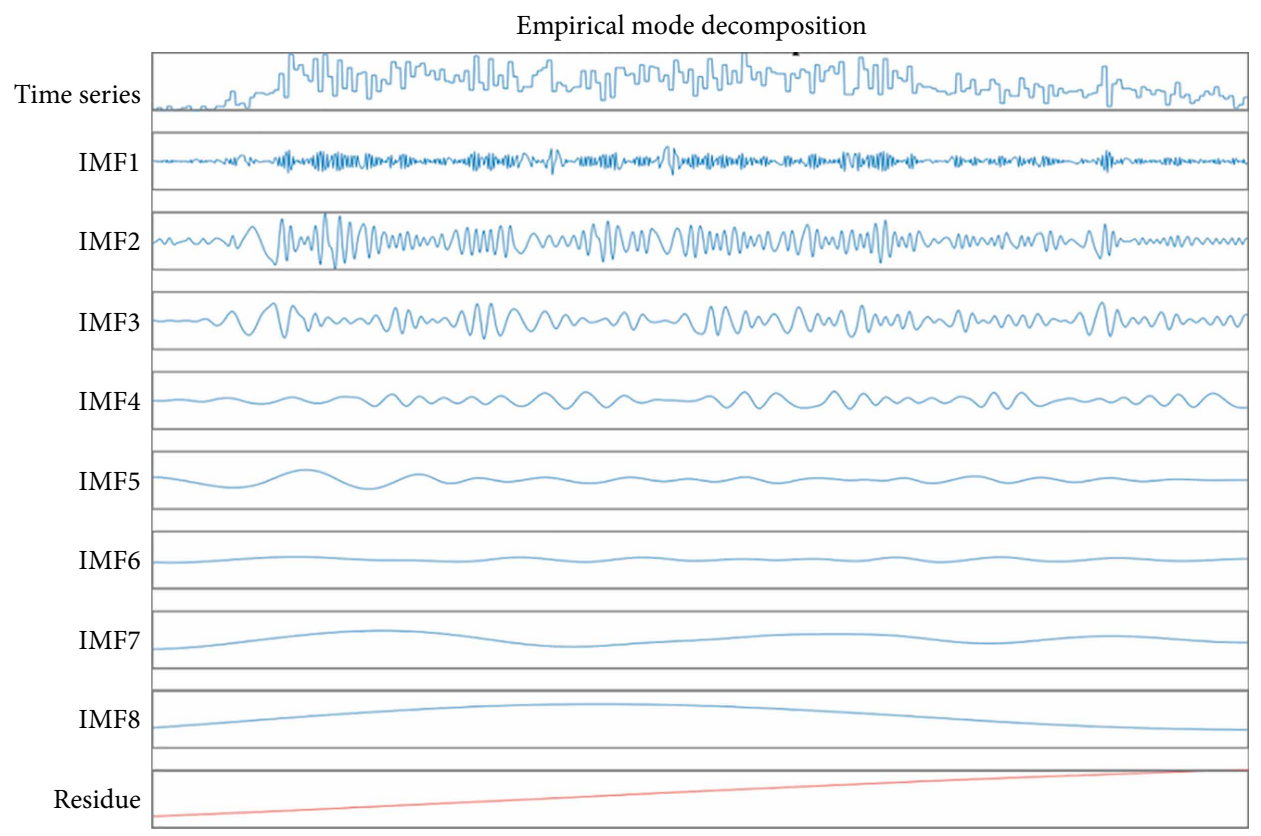

FIGURE 3: Raw traffic volume time series, corresponding IMFs, and residual.

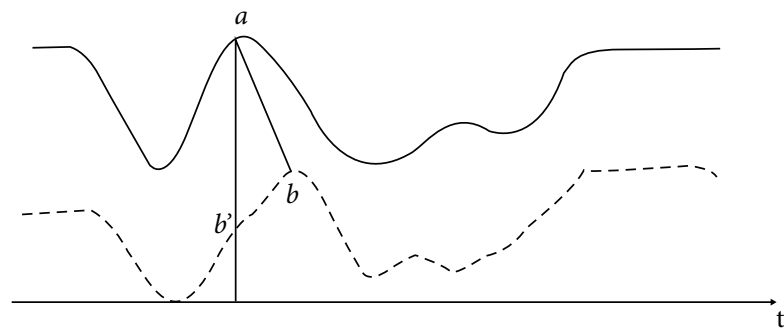

Figure 4: Difference between Euclidean and DTW distance.

point in time series $T(m)$ and the $j$-th point in time series $S(n)$. Note that matrix $D$ must be computed as the order of above equations, i.e. we must compute $D(1,1)$ first, then $D(i, 1)$, $D(1, j)$, and compute $D(i, j)$ at last. The minimum cumulative distance $D(m, n)$ is the target warping distance of two time series and the calculation path is the target warping path.

The distance between two time series $T$ and $S$ now can be easily calculated as follows:

$$
\text { DTW_dist }(T, S)=\sqrt{D(m, n)} .
$$

Because square root of the above equations is not important when comparing distances together, we can use this equation:

$$
\text { DTW_dist }(T, S)=D(m, n) .
$$

Moreover, to accelerate computational time and get a more accurate distance measure, some global constraints were proposed to DTW. For more details about DTW technique as well as its constraints, readers can refer to [29].

3.2.3. An Example. DTW distance calculation process is illustrated by the examples below. Suppose there are two time series as follows: $Q=(6,5,4,6,5,4,9,10)$ and $C=(5,4,4,5$,
TABlE 1: An example of a matrix cumulative distance of two time series.

\begin{tabular}{lcccccccc}
\hline$C$ & & & \multicolumn{7}{c}{$Q$} & & \\
& $\mathbf{6}$ & $\mathbf{5}$ & $\mathbf{4}$ & $\mathbf{6}$ & $\mathbf{5}$ & $\mathbf{4}$ & $\mathbf{9}$ & $\mathbf{1 0}$ \\
\hline $\mathbf{5}$ & $\mathbf{1}$ & $\mathbf{1}$ & 2 & 3 & 3 & 4 & 20 & 45 \\
$\mathbf{4}$ & 5 & 2 & $\mathbf{1}$ & 5 & 4 & 3 & 28 & 56 \\
$\mathbf{4}$ & 9 & 3 & $\mathbf{1}$ & 5 & 5 & 3 & 28 & 64 \\
$\mathbf{5}$ & 10 & 3 & 2 & $\mathbf{2}$ & 5 & 4 & 19 & 44 \\
$\mathbf{4}$ & 14 & 4 & 2 & 6 & $\mathbf{3}$ & 3 & 28 & 55 \\
$\mathbf{4}$ & 18 & 45 & 2 & 6 & 4 & $\mathbf{3}$ & 28 & 64 \\
$\mathbf{8}$ & 22 & 14 & 6 & 6 & 13 & 19 & $\mathbf{4}$ & 8 \\
$\mathbf{7}$ & 23 & 18 & 15 & 7 & 10 & 19 & $\mathbf{8}$ & $\mathbf{1 3}$ \\
\hline
\end{tabular}

Note: The bold values means the target distance calculation process and result.

$4,4,8,7)$. In order to calculate the distance between them, a matrix $D$ of cumulative distance was constructed (see Table 1) by Equation $(10) . D(m, n)=D(8,8)=13$ is the target warping distance between $Q$ and $C$, and the calculation path is the target warping path (see Table 1).

3.3. Fuzzy C-means (FCM) Clustering. Clustering is used to group $n$ points into $c$ clusters so that the within-cluster data are more similar to each other than to those in other clusters [30]. The advantage of clustering is to arrange a huge amount of data into several pieces and help users to identify the choice of interest. Clustering is a method using the criteria predefined to divide the input data into different clusters, and it helps to differentiate similarities and dissimilarities and compute useful inferences from them.

Fuzzy clustering is a form of soft clustering method, and each data point can belong to more than one cluster. The fuzzy C-means (FCM) clustering attempts to group a finite data set $X=\left\{x_{1}, x_{2}, \ldots, x_{n}\right\}$ into $c$ fuzzy clusters. $x_{i}$ represents feature 


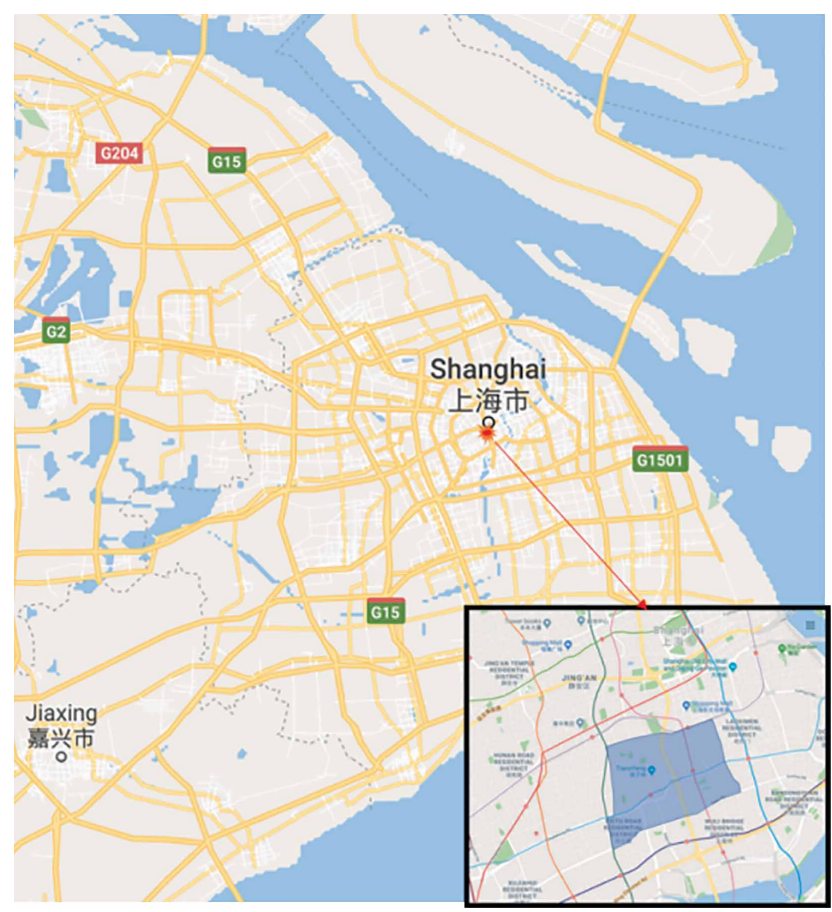

FIGURE 5: Location of research area in Shanghai, China.

vector. Given the data set and cluster number $c$, the algorithm returns cluster centres $C=\left\{c_{1}, c_{2}, \ldots c_{c}\right\}$ and a matrix of membership $U=u_{i j} \in[0,1], i=1, \ldots, n, j=1, \ldots c$, in which $\mu_{i j}$ means the membership degrees of $x_{i}$ to the cluster $c_{j}$.

$$
u_{i j}=\frac{1}{\sum_{k=1}^{c}\left(\left(x_{i}-c_{j}\right) /\left(x_{i}-c_{k}\right)\right)^{2 /(m-1)}},
$$

where $m$ is the fuzzification coefficient and it determines the fuzziness of the cluster. $m$ is a constant greater than 1 , and $m=2$ in this study [31].

For FCM, the centroid of a cluster is the mean of all points, weighted by their membership to the cluster.

$$
c_{j}=\frac{\sum_{i=1}^{n} u_{i j}^{m} \times x_{i}}{\sum_{i=1}^{n} u_{i j}^{m}} .
$$

The FCM aims to minimize the objective function:

$$
\arg \min _{C} \sum_{i=1}^{n} \sum_{j=1}^{c} u_{i j}^{m}\left|x_{i}-c_{j}\right|^{2} .
$$

The fuzzy C-means (FCM) clustering is composed of the following step:

Step 1. Given data set $X=\left\{x_{i}\right\}_{i=1}^{n}$, and cluster number $c$; Step 2. $U^{0}$ is randomly initialized;

Step 3. At $s$-th step: calculate the centres vectors $C^{(s)}=\left[c_{j}\right]$ with $U^{(s)}$, see Equation (14);

Step 4. Update $U^{(s)}$ with $C^{(s)}$ by Equation (13) into $U^{(s+1)}$;

Step 5. If $\left|U^{(s+1)}-U^{(s)}\right|<\varepsilon$, then STOP; otherwise, return to step 3.

\section{Data Collection and Description}

A downtown area with expressway on-ramp, located in Xuhui District, Shanghai, China (see Figure 5), is chosen as the study area. It consists of 43 intersections and an expressway on-ramp, and the on-ramp is located between intersection \#19 and \#28 (see Figure 6). The roads with red mark are arterial roads, and the detailed information of road type is shown in Table 2.

The traffic data are collected on $7^{\text {th }}$ March, 2017 by inductive detection loops of SCATS control system, including the traffic volume time series. Inductive detection loops are located on every approach of intersections and the data was collected per cycle shown in Table 3. In Table 3, the first column is the data acquisition time, the second column is the serial number of inductive detection loop. The third column is the phase of signal light. The fourth and fifth column mean the length and max length of current phase. The last column represents the traffic volume in this phase. In data processing, the original data is divided into every second and then aggregated into every minute.

The SCATS system also provides other information of each intersection. Take one intersection (\#39) as an example. It is shown in Figure 7 that the locations of inductive detection loops, the lane function and the phases information of signal are marked. Apart from intersection phasing and timing information and inductive detection loop data, the SCATS system can also provide control strategy of intersections, such as coordinated control among several intersections.

\section{Model Results}

5.1. EMD Filtering Method Result. EMD method is used to filter the original data of approaches at every intersection. Take \#28 intersection as an example, the traffic volume time series is decomposed (see Figure 3). In the reconstruction of time series, the calculation based on Equation (7) shows that $j$ equals to 5 . Then the reconstruction of time series equals to the sum of IMF5-IMF8 and residue (see Figure 8). It can be seen that EMD method can smooth time series and maintain the feature of original data.

\subsection{Calculation Result of DTW Distance between Each} Intersection Approach and On-Ramp. After data processing, denoised traffic volume time series of left-turn, straight and rightturn approaches of 43 intersections are obtained. The DTW distances between traffic volume time series of each approaches and on-ramp are compared. Take the \#5 intersection in Figure 6 as an example: it has left-turn, straight and right-turn lanes at the eastern, western, southern and northern approaches. Assuming drivers do not make a detour to the on-ramp, so the valid routes from \#5 intersection to on-ramp only contain the straight and right-turn lanes at the northern approach and the straight and left-turn lanes at the eastern approach. Based on this assumption, all valid routes to on-ramp have been screened out, and we can obtain 124 intersection approaches traffic volume time series. Finally, DTW distances between the traffic volume time series at selected approaches and onramp are calculated. In order to explore how DTW distances 


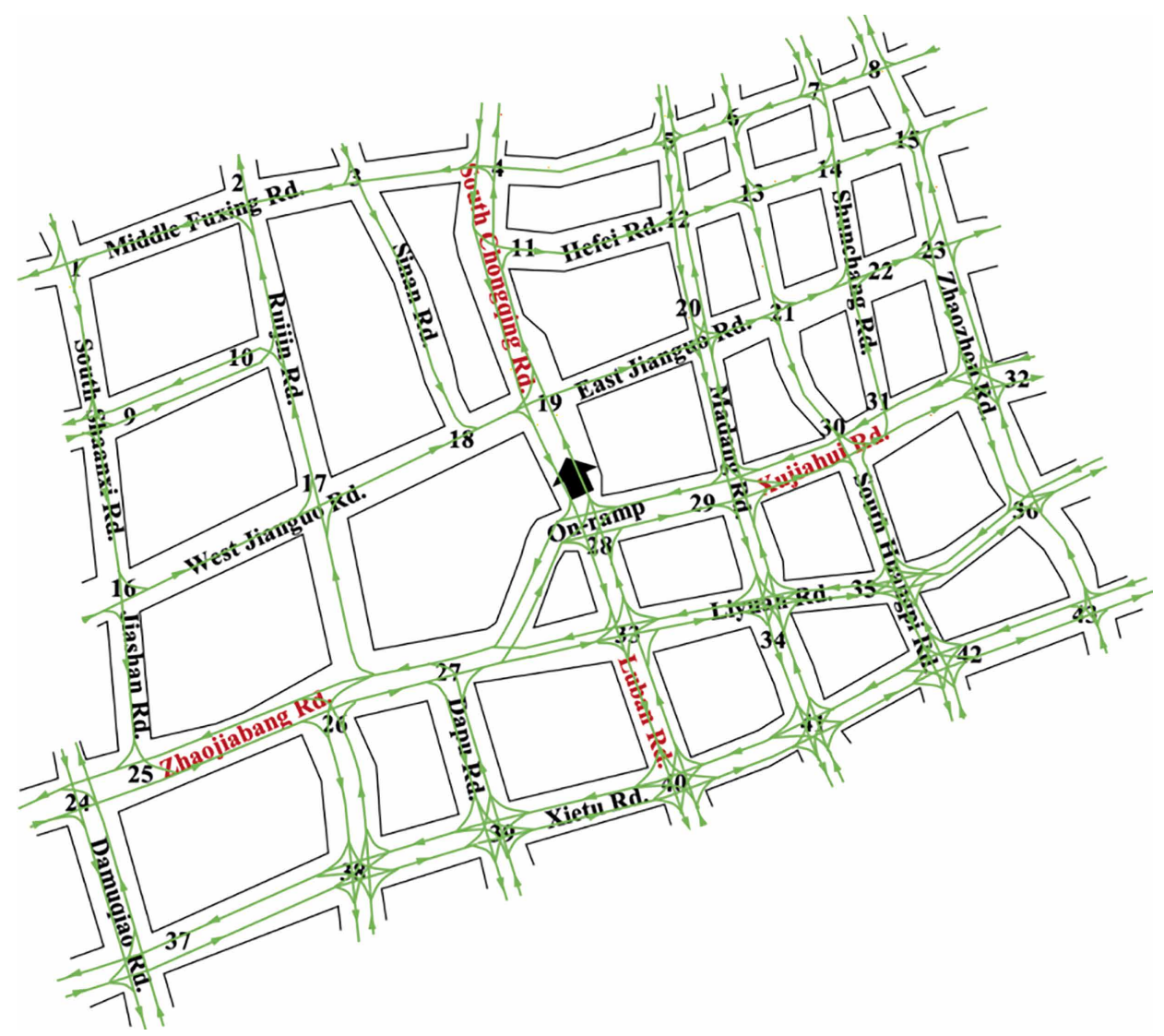

FIGURE 6: Layout and number of intersections and on-ramp.

TABLE 2: Road information of network.

\begin{tabular}{|c|c|c|c|c|c|}
\hline Road & Number of lanes & Road type & Road & Number of lanes & Road type \\
\hline \multicolumn{6}{|l|}{ East-west direction } \\
\hline $\begin{array}{l}\text { Middle Fuxing Rd. } \\
(\# 8 \rightarrow \# 1)\end{array}$ & 2 (one-way) & Branch road & $\begin{array}{l}\text { Zhaojiabang Rd. } \\
\quad(\# 24 \rightarrow \# 33)\end{array}$ & 10 (two-way) & Arterial road \\
\hline 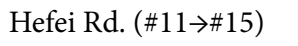 & 2 (one-way) & Branch road & Liyuan Rd. (\#33 $\rightarrow \# 36)$ & 2 (two-way) & Branch road \\
\hline Jianguo Rd. (\#16 $\rightarrow \# 23)$ & 2 (one-way) & Branch road & Xietu Rd. (\#37 $\rightarrow \# 43)$ & 6 (two-way) & Secondary trunk road \\
\hline Xuhui Rd. (\#28 $\rightarrow \# 32)$ & 10 (two-way) & Arterial road & - & - & - \\
\hline \multicolumn{6}{|l|}{ South-north direction } \\
\hline $\begin{array}{l}\text { Da Muqiao Rd. } \\
(\# 24 \rightarrow \# 37)\end{array}$ & 4 (two-way) & Secondary trunk road & Luban Rd. (\#40 $\rightarrow \# 38)$ & 12 (two-way) & Arterial road \\
\hline Shanxi Rd. (\#1 $\rightarrow \# 16)$ & 1 (one-way) & Branch road & $\begin{array}{l}\text { Madang Rd. } \\
(\# 5 \rightarrow \# 41)\end{array}$ & 2 (two-way) & Secondary trunk road \\
\hline Jiashan Rd. (\#16 $\rightarrow \# 25)$ & 1 (one-way) & Branch road & $\begin{array}{l}\text { South Huangpi Rd. } \\
\qquad(\# 6 \rightarrow \# 42)\end{array}$ & 5 (two-way) & Secondary trunk road \\
\hline Ruijin Rd. (\#2 $\rightarrow \# 17)$ & 1 (one-way) & Branch road & $\begin{array}{l}\text { Shunchang Rd. } \\
\qquad(\# 7 \rightarrow \# 31)\end{array}$ & 2 (one-way) & Branch road \\
\hline Sinan Rd. $(\# 3 \rightarrow \# 18)$ & 1 (one-way) & Branch road & $\begin{array}{l}\text { Zhaozhou Rd. } \\
\qquad(\# 8 \rightarrow \# 43)\end{array}$ & 2 (two-way) & Branch road \\
\hline $\begin{array}{l}\text { South Chongqing Rd. } \\
(\# 4 \rightarrow \# 28)\end{array}$ & 12 (two-way) & Arterial road & - & - & - \\
\hline
\end{tabular}

vary in different periods of one day, a day is divided into five periods: 00:00-06:00 (off peak), 06:00-09:00 (morning peak), 09:00-16:00 (off peak), 16:00-19:00 (evening peak), and 19:00-24:00 (off peak).
According to calculation results, the top five intersection approaches with highest similarity during 06:00-09:00 are shown in Figure 9. It is obvious that there is a similar trend of traffic volume change at the on-ramp and intersection approaches. 
TABLE 3: Example of inductive detection loop data.

\begin{tabular}{|c|c|c|c|c|c|}
\hline Start time & Detectorid & Phasic & Phasic length (s) & Max length (s) & Flow (vehicle) \\
\hline 2017/3/1 0:00 & 24007 & $\mathrm{C}$ & 42 & 111 & 3 \\
\hline 2017/3/1 0:00 & 24008 & $\mathrm{C}$ & 42 & 111 & 3 \\
\hline $2017 / 3 / 10: 00$ & 24001 & A & 48 & 110 & 3 \\
\hline 2017/3/1 0:00 & 24002 & A & 48 & 110 & 0 \\
\hline 2017/3/1 0:00 & 24004 & A & 48 & 110 & 2 \\
\hline 2017/3/1 0:00 & 24005 & A & 48 & 110 & 4 \\
\hline 2017/3/1 0:01 & 24003 & B & 20 & 110 & 0 \\
\hline 2017/3/1 0:01 & 24006 & B & 20 & 110 & 0 \\
\hline 2017/3/1 0:01 & 24007 & $\mathrm{C}$ & 42 & 110 & 5 \\
\hline 2017/3/1 0:01 & 24008 & $\mathrm{C}$ & 42 & 110 & 3 \\
\hline
\end{tabular}

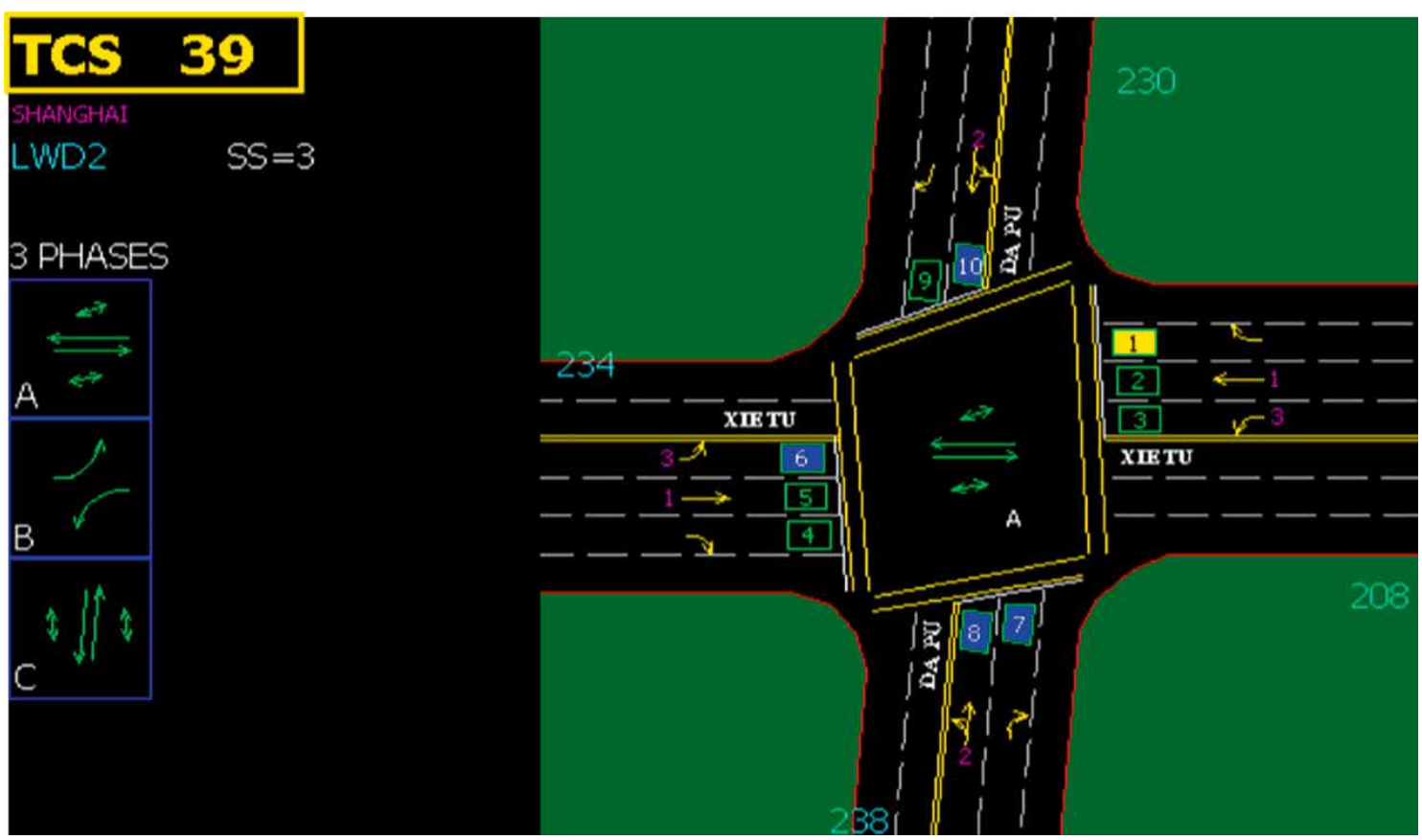

FIGURE 7: Information map of one intersection in SCATS system.

Figure 10 shows the intersection approaches with 15 maximum DTW values and 15 minimum DTW values. Larger DTW value means lower similarity degree. It can be found that (1) in general, intersections close to on-ramp is highly similar with on-ramp, such as \#19, \#28 intersection (see Figure 6). But there are exceptions, some intersection approaches far away from on-ramp also have high similarity, such as \#25 and \#24 intersection. It means there are more traffic flows choosing these routes to enter the ramp; (2) The location of intersection does not play a decisive role. For the similarity degree between intersection approaches and on-ramp, for example, \#38 intersection (see Figure 6) is not located on an arterial road, but it shows a high similarity with on-ramp.

5.3. Clustering Result of Intersections Approaches. To achieve critical routes identification, various factors are considered. After comparing the clustering effects and efficiency, three parameters are selected in this work. A three-dimensional feature vector is built for every intersection approach traffic flow, and three features are DTW value between time series of intersection approaches and on-ramp, space distance between intersection and on-ramp and traffic volume of intersection approaches. Then three feature factors should be normalized to $[0,1]$ and the fuzzy $\mathrm{C}$-means clustering algorithm is used to cluster all intersection approaches. Clustering result in five periods on $7^{\text {th }}$ March, 2017 is displayed in Figure 11.

Table 4 describes the results of univariate analysis using $F$-test based on three clustering groups at different periods. In Table 4, it can be seen, in different time periods, the DTW, traffic volume and distance are all significantly different in three groups (sig. <0.05). It means this clustering method can effectively classify routes among intersections relating to DTW, traffic volume and distance.

According to the clustering result, intersection approaches in research area are clustered into three classifications in different periods. In the first classification, approaches with the smallest DTW means the highest similarity to on-ramp, and they are the critical routes in this period. These routes need 


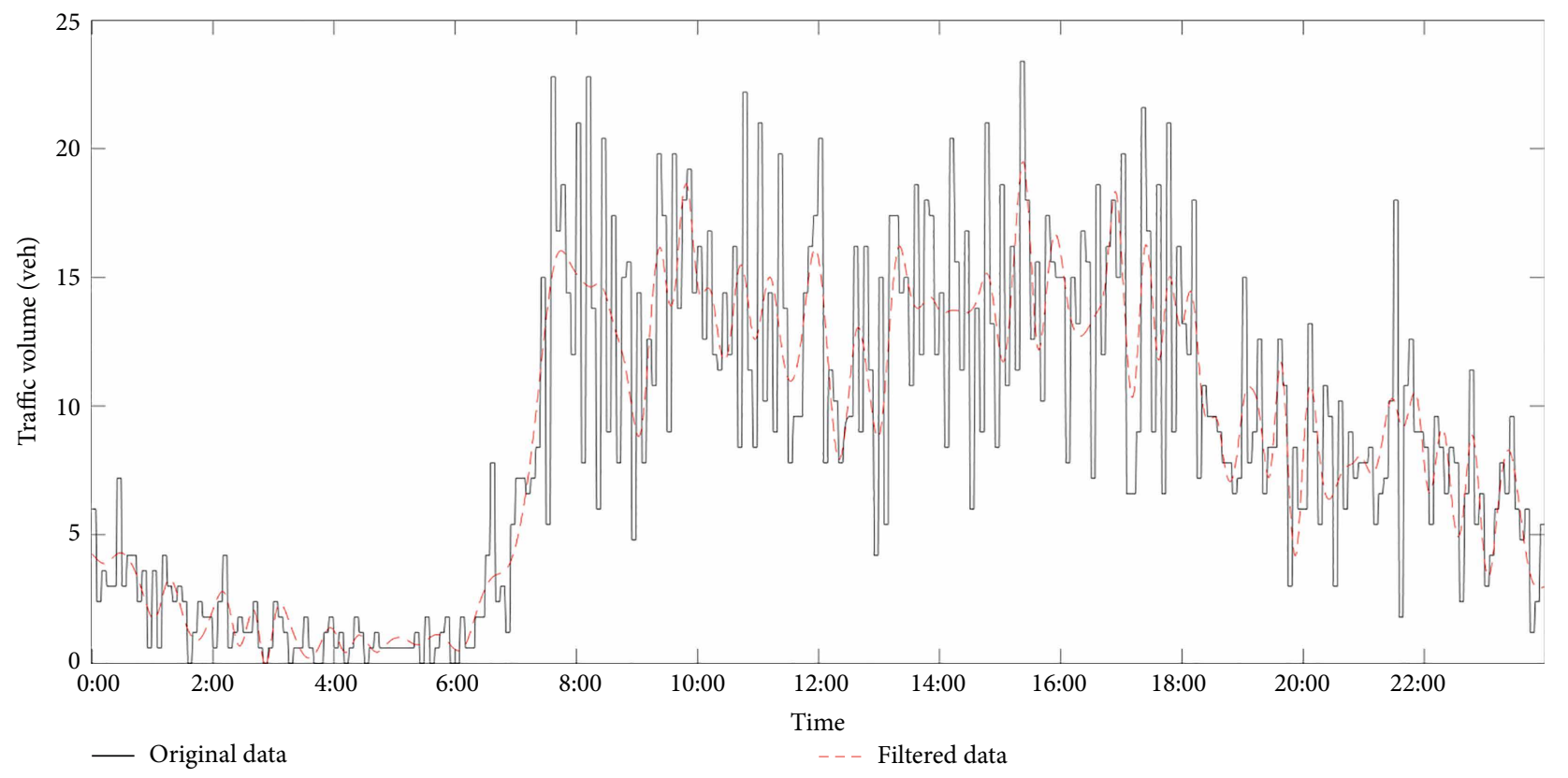

FIGURE 8: Contrast between original traffic flow data and EMD de-noising data.

TABLE 4: Significance testing of interval duration in different groups.

\begin{tabular}{lcccc}
\hline Time period & Feature factor & Df & $F$-value & Significance \\
\hline \multirow{3}{*}{ 1 (00:00-06:00) } & DTW & 2 & 281.69 & $2.9700 \mathrm{e}-46$ \\
& Traffic volume & 2 & 57.96 & $2.2087 \mathrm{e}-18$ \\
& Distance & 2 & 14.31 & $2.6372 \mathrm{e}-06$ \\
2 (06:00-09:00) & DTW & 2 & 113.08 & $7.5765 \mathrm{e}-29$ \\
& Draffic volume & 2 & 94.95 & $7.1087 \mathrm{e}-26$ \\
& Distance & 2 & 10.28 & $7.2876 \mathrm{e}-05$ \\
3 (09:00-16:00) & DTW & 2 & 17144 & $4.9083 \mathrm{e}-36$ \\
& Distfic volume & 2 & 128.53 & $1.1657 \mathrm{e}-30$ \\
& DTW & 2 & 257.91 & $2.2827 \mathrm{e}-47$ \\
4 (16:00-19:00) & Traffic volume & 2 & 186.14 & $1.1925 \mathrm{e}-37$ \\
& Distance & 2 & 14.69 & $1.9370 \mathrm{e}-06$ \\
& DTW & 2 & 369.55 & $2.9387 \mathrm{e}-52$ \\
5 (19:00-24:00) & Traffic volume & 2 & 278.19 & $5.5307 \mathrm{e}-46$ \\
& Distance & 2 & 3.61 & 0.0301 \\
\hline
\end{tabular}

Note: $D f$ means Degree of freedom, $F$ means the difference among different groups, Significance means the significance level.

coordinated control with on-ramp. The approaches in the second classification are with medium DTW values. When the control of routes in the first classification is achieved, routes in the second classification can also be coordinately controlled if the traffic service level is still low and control condition allows it; the third classification has the lowest similarity with on-ramp, and no special control is needed for this classification. According to the clustering results of 5 periods in one day, the priority level of the routes is obtained (see Figure 12). The clustering results show that different control strategies should be adopted in different periods of one day. It can be seen that the coordinated control results in different periods always contain the most and even the whole arterial roads, while only several nontrunk roads need coordinated control. For example, in Figure 12(a), the critical route identification result coincides with arterial roads in South Chongqing Rd., Zhaojiabang Rd. and Xujiahui Rd. Although Luban Rd. is not contained in critical routes, we can see that the critical routes turn from \#43, \#42, \#41 on nontrunk road to \#33 on arterial road. Thus, it can be seen that the coordinated control of on-ramp and intersections can not be limited in the nearest intersection to on-ramp, and the conventional control of arterial roads can not always lead to the best effect of traffic flow from intersections to on-ramp.

\section{Effect Validation}

After the identification of critical routes in different periods of a day with the proposed method, we can test the effectiveness of this method with TransModeler simulation.

6.1. Micro-Simulation Model. The proposed critical routebased control strategy is tested and evaluated using the scalable, high-performance microscopic simulation package of TransModeler software. In order to accurately code the intersections in TransModeler, large scale background images of the studied intersections and on-ramp are needed. For each link, several specifications are defined in TransModeler: (1) number of lanes for segment; (2) lane width and distance; (3) signal phases; (4) speed limit; (5) input volume. The network in TransModeler is constructed as Figure 6.

After the network coding, network calibration and validation are required for a reliable micro-simulation study. The simulation uses the collected traffic demands of five periods on $7^{\text {th }}$ March, 2017. Five periods are chosen as the simulation periods, and every period was simulated for $3 \mathrm{~h}$ and $20 \mathrm{~min}$. The first $20 \mathrm{~min}$ is considered to be the warm-up period for 


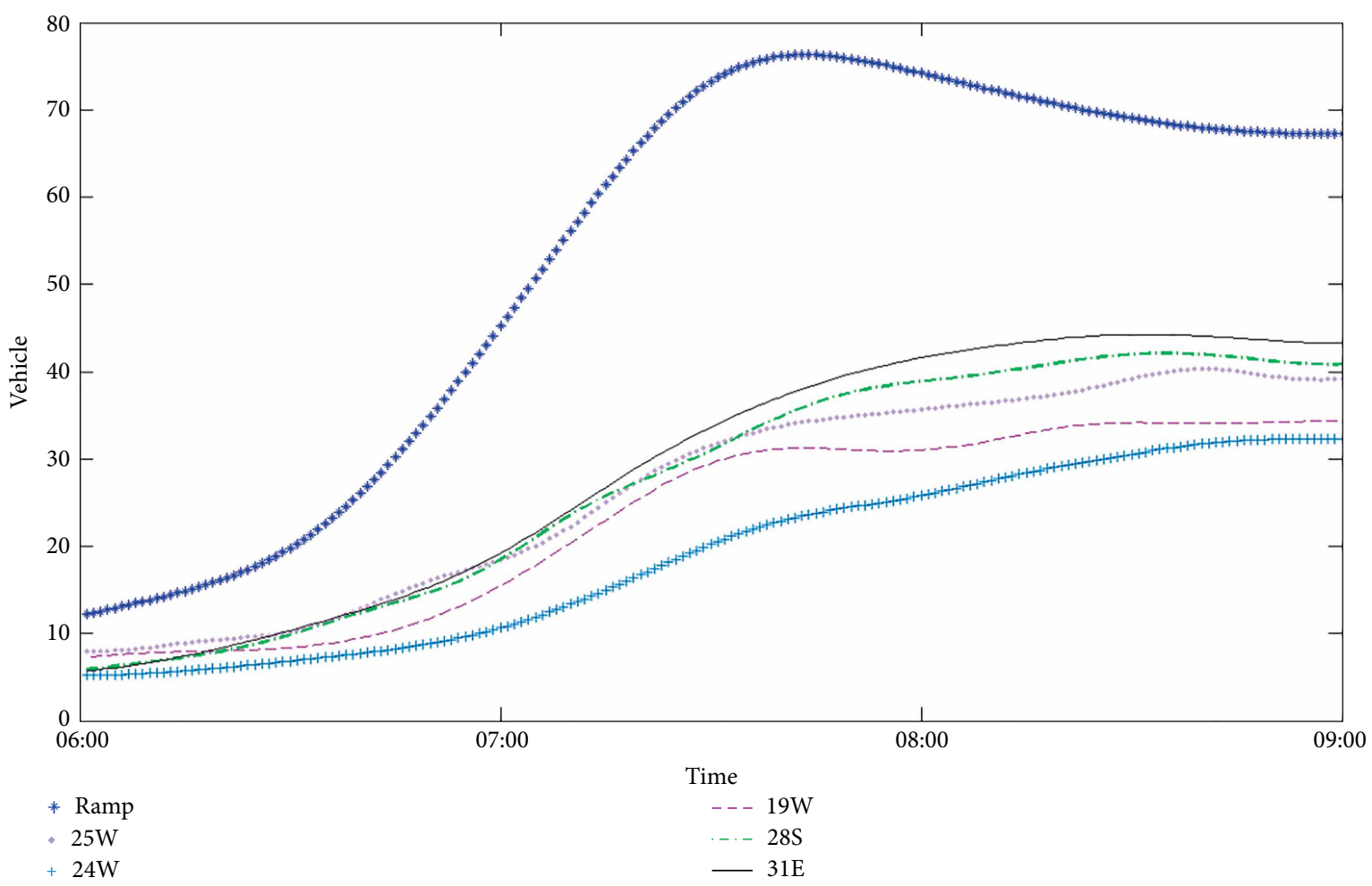

FIgURE 9: Traffic volume at on-ramp and 5 most similar intersection approaches at 06:00-09:00.

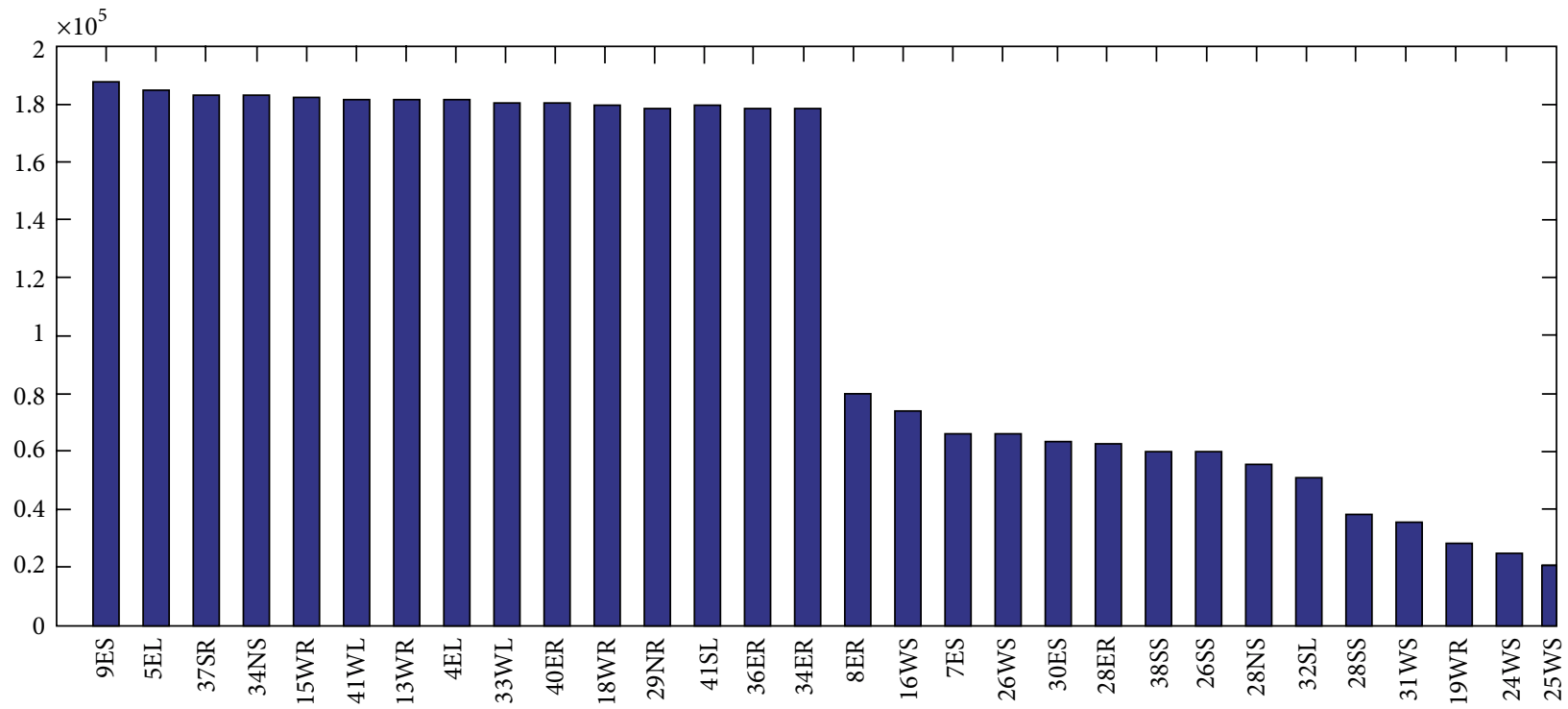

FIGURE 10: Distribution of intersection approaches with Maximum 15 and Minimum 15 DTW. Note: E-east; W-west; S-south; N-north.

vehicles to fill in the network, because it takes less than $20 \mathrm{~min}$ to finish the longest trip in the network in the real world. The simulation model is calibrated for traffic volume at 5 -min intervals. The simulation can be calibrated and validated by evaluating the Geoffrey E. Havers (GEH) equation [32]. GEH is a valid volume calibration parameter, and the definition is as follows:

$$
\mathrm{GEH}=\sqrt{\frac{(E-V)^{2}}{(E+V) / 2}}
$$

where $E=$ modelled volume (vph) is the simulated volumes obtained from the simulation network, and $V=$ field volume (vph) is the observed volume of in-field detectors. The simulated traffic volumes are aggregated to 5-min intervals. The GEH statistics are from two selected detectors at the west and east approaches of \#28 intersection for a $3 \mathrm{~h} \mathrm{sim-}$ ulation period (see Table 5). The results from 10 simulation runs show that $94.3 \%$ of the observed GEHs are less than 5 for all intersections, which means the network setup in 


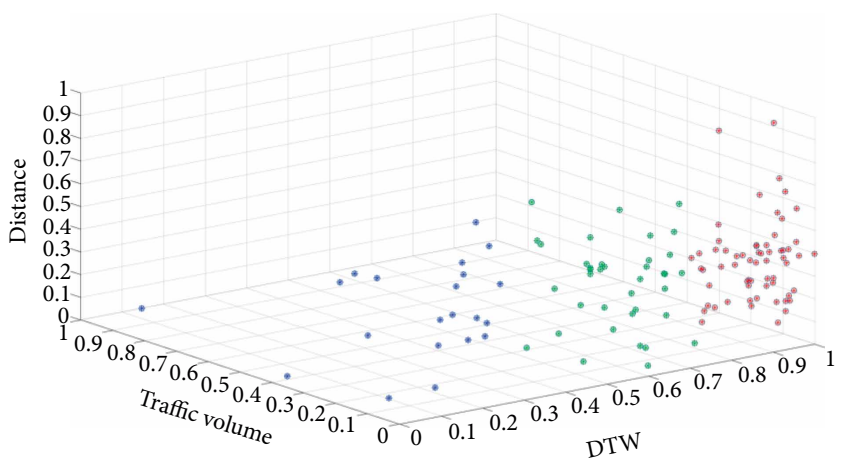

(a)

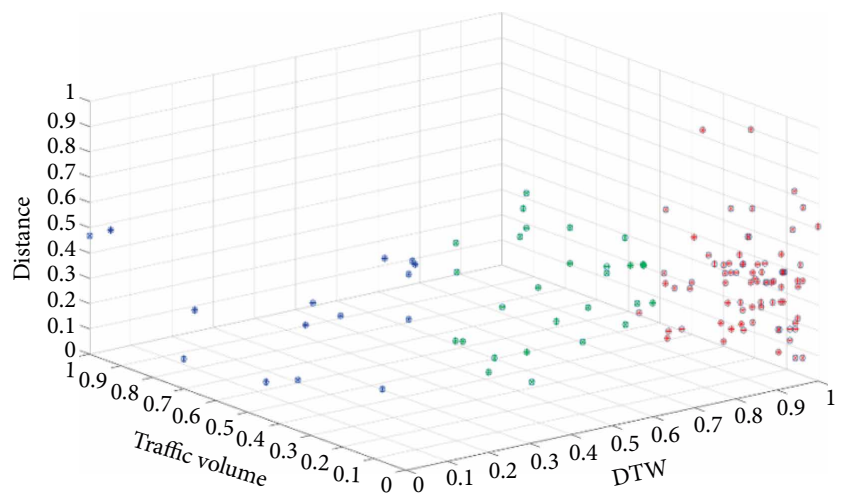

(c)

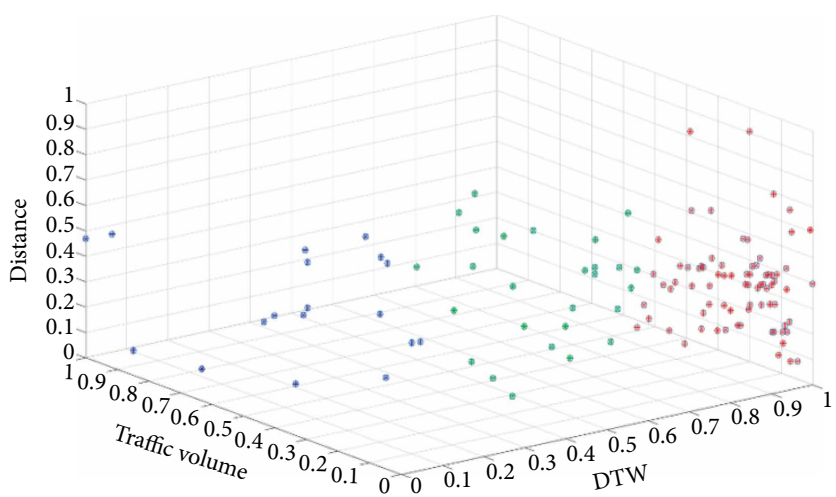

(b)

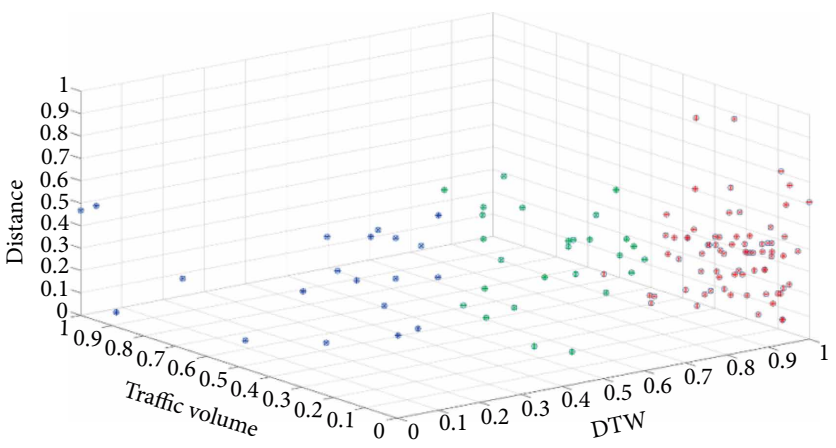

(d)

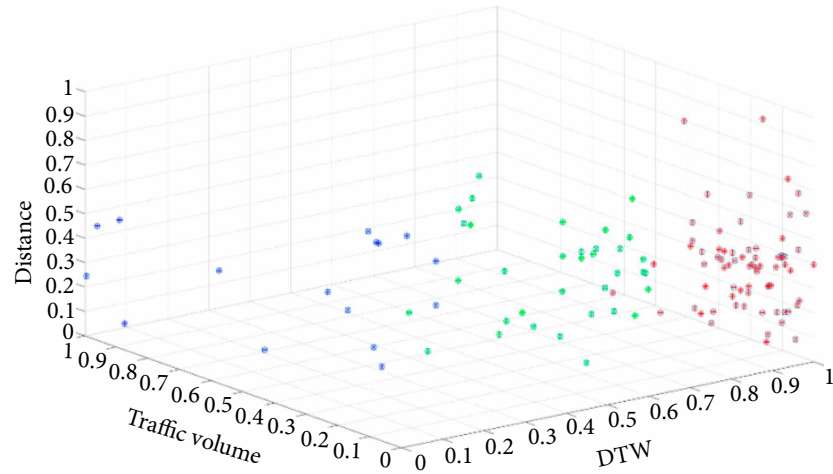

(e)

FIGURE 11: Data clustering results of each intersection approaches in different periods of one day. (a) 00:00-06:00, (b) 06:00-09:00, (c) 09:00-16:00, (d) 16:00-19:00, and (e) 19:00-24:00.

TransModeler can accurately represent the intersections traffic flow characteristics.

6.2. Control Strategy. Critical route control strategy (CC) proposed in this paper and arterial road control strategy (AC) are explored in simulation. No control strategy (NC) and AC are used as references to the proposed CC. NC means the actual state of intersections in study area. AC means achieving green wave control on arterial roads. CC is a coordinated control strategy with green waves for intersections on critical routes. Green wave control strategy is based on Webster formula. Firstly, calculate and determine the isolated intersection timing and phasing scheme and obtain the common cycle length of arterial roads or critical routes. Then, the phase differences between the intersections on arterial roads or critical routes are calculated by numerical method. Thus, the timing scheme of coordinated control of the arterial roads or critical routes are obtained.

Take AC as an example, after the calculation, the \#28 intersection signal cycle is set to $120 \mathrm{~s}$, and it's the maximum cycle of arterial roads intersections. Therefore, the system cycle length is set to $120 \mathrm{~s}$. Then green signal ratio is determined by actual traffic conditions of each intersection, and phase difference is determined by average speed and length of the road section. According to these control parameters to achieve simulation. 


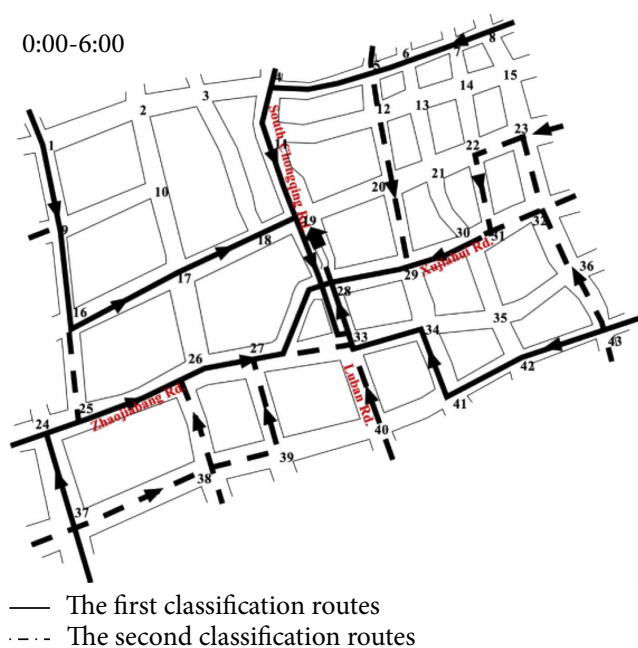

(a)

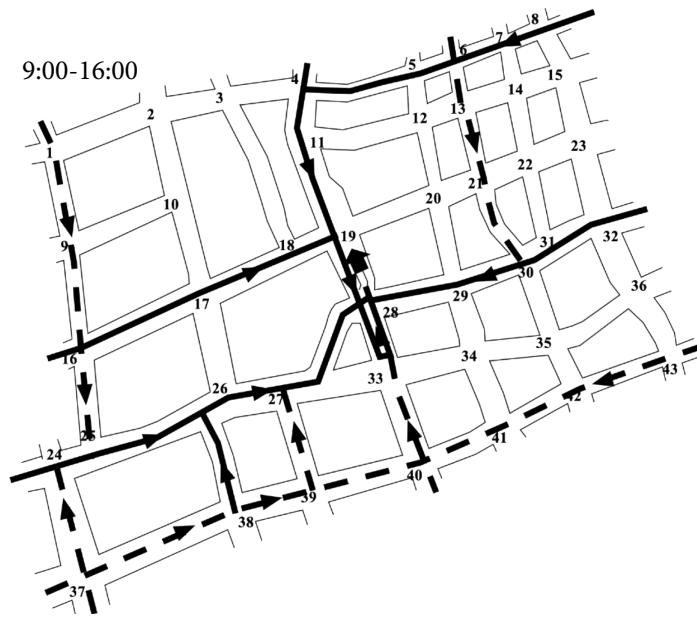

- The first classification routes

...- The second classification routes

(c)

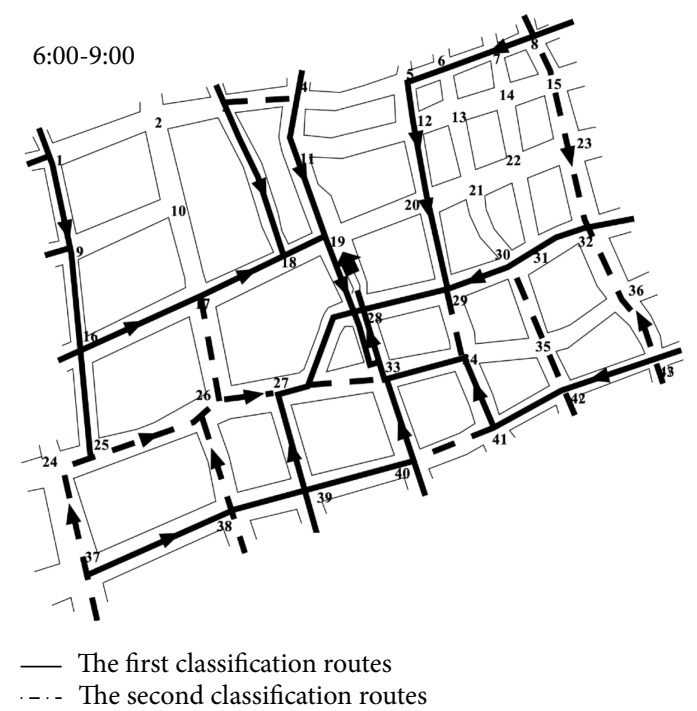

(b)

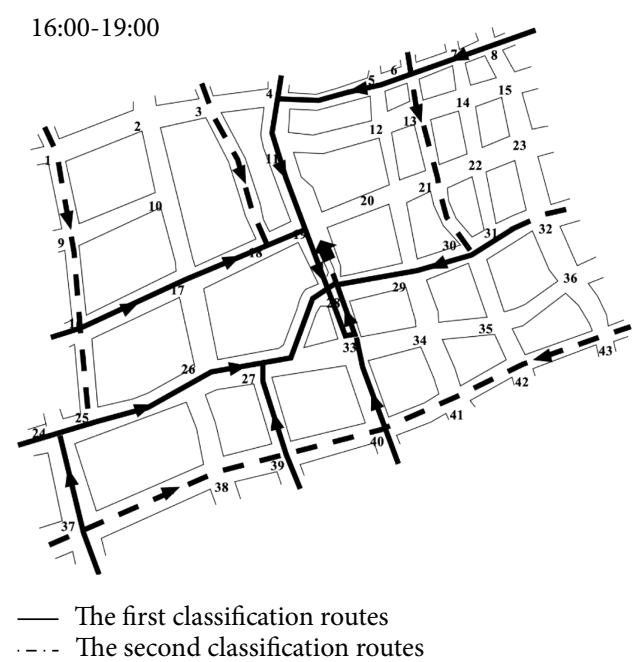

(d)

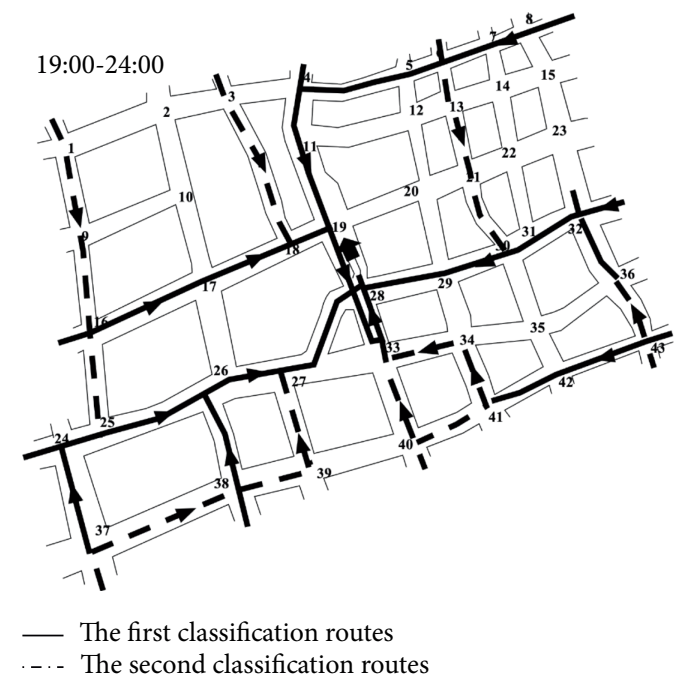

(e)

Figure 12: Routes identification results in different periods of one day. (a) 00:00-06:00, (b) 06:00-09:00, (c) 09:00-16:00, (d) 16:00-19:00, and (e) 19:00-24:00. 
TABLE 5: GEH statistics for the two calibrated locations.

\begin{tabular}{lccccc}
\hline Location & $N$ & Mean & Std. dev. & Minimum & Maximum \\
\hline West approach of \#28 intersection & $36^{\mathrm{a}}$ & 2.47 & 1.73 & 0.11 & 5.55 \\
East approach of \#28 intersection & 36 & 2.86 & 1.35 & 1.18 & 5.43 \\
\hline
\end{tabular}

${ }^{a}$ Number of observations, $12 * 3=365$-min intervals per hour.

TABLE 6: Weighted average delay of the whole area(s).

\begin{tabular}{lccccc}
\hline \multirow{2}{*}{ Control strategy } & \multicolumn{4}{c}{ Time period } \\
& $00: 00-06: 00$ & $06: 00-09: 00$ & $09: 00-16: 00$ & $16: 00-19: 00$ & $19: 00-24: 00$ \\
\hline NC & 12.1 & 30.9 & 19.1 & 29.2 & 22.4 \\
AC & 11.4 & 28.4 & 18.4 & 26.7 & 20.8 \\
AC improvement compared to NC & $5.78 \%$ & $8.09 \%$ & $3.66 \%$ & $8.56 \%$ & $7.14 \%$ \\
CC & 11.5 & 27.7 & 17.8 & 26.3 & 20.3 \\
CC improvement compared to NC & $4.96 \%$ & $10.36 \%$ & $6.81 \%$ & $9.93 \%$ & $9.38 \%$ \\
\hline
\end{tabular}

TABLE 7: Controlled intersections of AC and CC control strategy.

\begin{tabular}{lr}
\hline Control strategy & Controlled intersections \\
\hline AC & $\# 4, \# 11, \# 19, \# 28, \# 33, \# 40, \# 24-\# 32$ \\
CC & $\# 1-\# 9-\# 16-\# 25 ; \# 16-\# 17-\# 18-\# 19 ; \# 8-\# 7-\# 6-\# 5 ; \# 5-\# 12-\# 20-\# 29 ; \# 32-\# 31-\# 30-\# 29-\# 28 ; \# 37-\# 38-\# 39-\# 40-\# 33-\# 28 ;$ \\
& $\# 39-\# 27-\# 28 ; \# 43-\# 42-\# 41-\# 34-\# 33$ \\
\hline
\end{tabular}

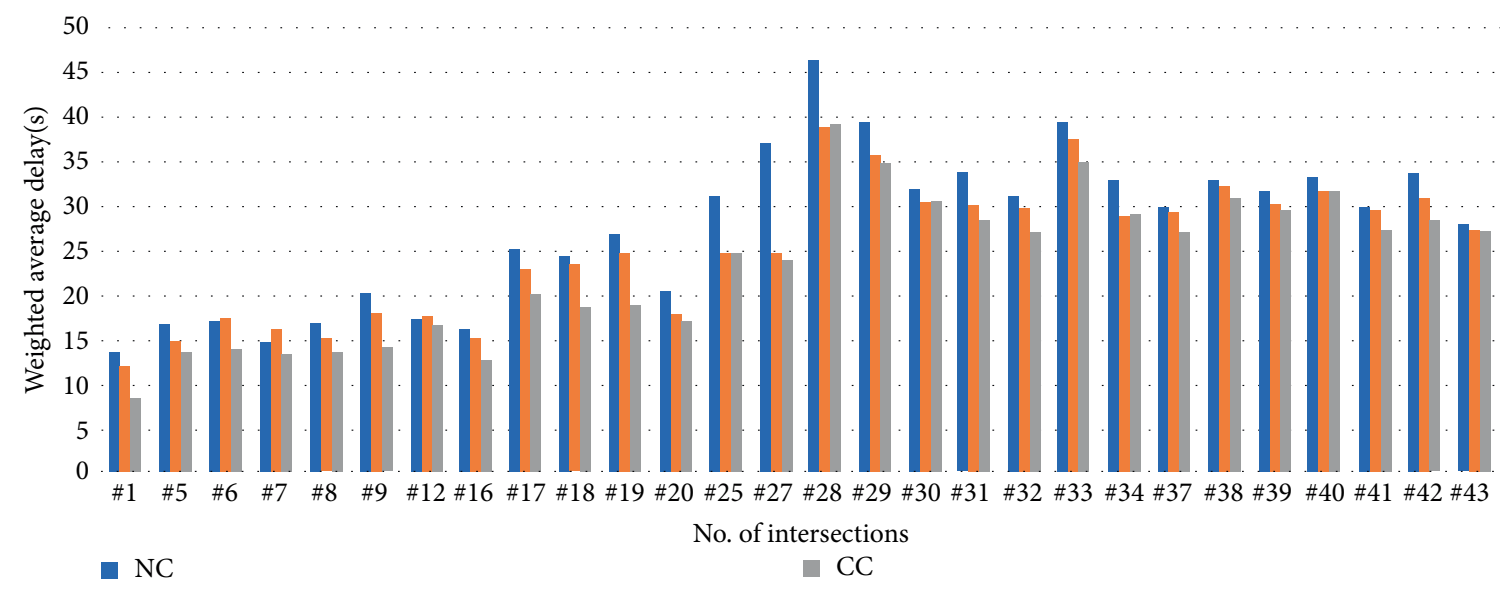

(a)

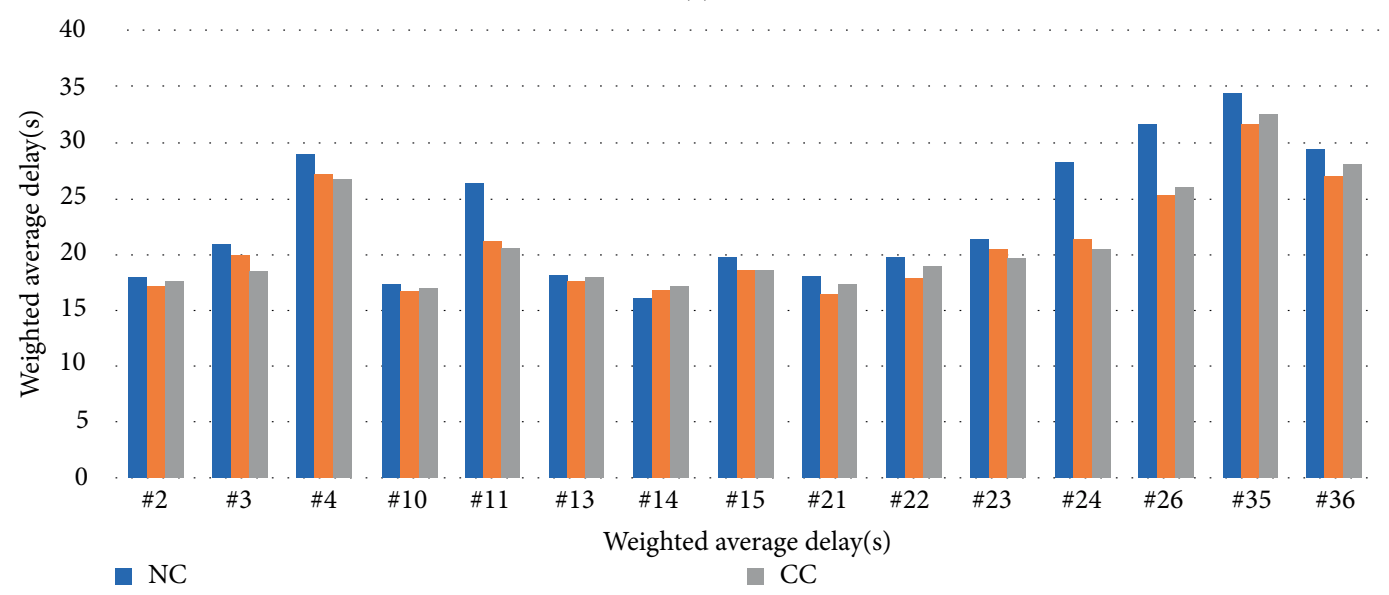

(b)

FIGURE 13: Weighted average delay in study area with different control strategies. (a) Intersections coordinately controlled by CC and (b) Intersections not coordinately controlled by CC. 
6.3. Simulation Result. Simulation result of 5 periods on $7^{\text {th }}$ March 2017 with TransModeler is shown in Table 6. The weighted average delay per vehicle is chosen as an indicator of intersection efficiency, and the weighted average delay is calculated as Equations (17) and (18) based on the flow of each intersection. It can be seen that Table 6 shows at most times, CC outperforms AC. And this is more obvious during peak period (06:00-09:00 and 16:00-19:00), which means CC has better effect with higher traffic demands on the whole.

$$
W A D_{j}=\frac{\sum_{i} V_{i j} * A D_{i j}}{\sum_{i} V_{i j}},
$$

where $W A D_{j}$ means the weighted average delay of the $j$-th intersection. $V_{i j}$ means the traffic flow measured at $i$-th approach at $j$-th intersection. $A D_{i j}$ means the average delay of the traffic flow measured at $i$-th approach at $j$-th intersection.

$$
W A D=\frac{\sum_{j} V_{j} * W A D_{j}}{\sum_{j} V_{j}},
$$

where $W A D$ means weighted average delay of the whole area; $V_{j}$ means traffic flow at the $j$-th intersection.

Take the morning peak (06:00-09:00) as an example. The critical route identification results shown in Figure 12(b) is simulated using coordinated control of the $\mathrm{NC}, \mathrm{AC}$ and $\mathrm{CC}$ strategy. The controlled intersections of AC and CC control strategy are shown in Table 7. Result of the weighted average delay at every intersection during the morning peak (06:0009:00) from 10 simulations is shown in Figure 13. Figure 13(a) shows the weighted average delay at the intersections which CC coordinately controls. It can be seen that CC has a more efficient control at these intersections. Figure 13(b) shows the weighted average delay at the intersections which CC doesn't control. It can be seen that for these intersections which are not controlled with CC, the performance does not necessarily get better or worse. But on the whole research area, CC is $10.36 \%$ more efficient than AC (see Table 6). It can be seen from Table 7 that more intersections are controlled, and the control effect is better in CC case than AC case.

\section{Conclusions and Discussion}

Traditional coordinated control strategy including subarea and arterial road control cannot achieve the traffic control among intersections and on-ramp. This paper has proposed a route identification method for on-ramp traffic and adjacent intersections. Compared with previous reviews of coordinated control of on-ramp and intersections, the paper is unique in that it provides a new perspective to use DTW distance to measure similarity between intersections and on-ramp and classify routes among intersections based on this.

We use a data-driven EMD method to denoise real-world traffic volume data, due to the widely-used wavelet filtering needs to take efforts to select an appropriate wavelet to achieve the best result [33]. Then an appropriate and innovative DTW distance is chosen to measure similarity between intersections and on-ramp, and it has considered the lag between upstream and downstream traffic flow. After this, the correlation degree between on-ramp and intersection approaches can be calculated. Then the clustering method are used to classify intersection approaches into three groups and to identify the critical routes. Finally, the validation of the critical route control is conducted with simulation test to compare effects of different control strategies. Compared to arterial road control strategy, critical route control strategy is more effective.

The similarity of two time series measured with DTW is a novel way in traffic control area, and this paper is just a start. In this paper, only the relationship between on-ramp and intersections is considered, and off-ramp will be considered in the future. For the selection of critical routes, it's an important task to determine the border of "green wave" control, and this is the aim of future work. In addition, only traffic volume was considered as one-dimension traffic feature in this paper. In the future, a multi-dimension index, which contains not only traffic volume but also speed and density, may be better to represent the traffic state before using DTW to explore the traffic characteristics. Then it can be more accurate to identify the bottleneck points in the network and give more helpful reference for coordinated control.

\section{Data Availability}

The funding agency does not allow the data to be open to the public without their permission.

\section{Conflicts of Interest}

The authors declare that they have no conflicts of interest.

\section{Acknowledgments}

The research is supported jointly by the National Natural Science Foundation of China (No. 71804127) and the Shanghai Science and Technology Committee (STCSM, Grant No. 18DZ1200200).

\section{References}

[1] Booze, Integrated Corridor Traffic Management Final Evaluation, Minnesota Department of Transportation, USA, 2000.

[2] M. Schmitt, C. Ramesh, and J. Lygeros, "Sufficient optimality conditions for distributed, nonpredictive ramp metering in the monotonic cell transmission model," Transportation Research Part B Methodological, vol. 105, pp. 401-422, 2016.

[3] T. Djukic, G. Flotterod, H. Van Lint, and S. Hoogendoorn, "Efficient real time OD matrix estimation based on principal component analysis," in International IEEE Conference on Intelligent Transportation Systems, pp. 115-121, 2012.

[4] L. Zhang, G. Chen, and T. Zhang, "Study on area coordination control system based on traffic state discrimination," in International Congress on Image \& Signal Processing, IEEE, 2017.

[5] Y. Bie, D. Wang, and X. Qu, "Modelling correlation degree between two adjacent signalised intersections for dynamic 
subarea partition," IET Intelligent Transport Systems, vol. 7, no. 1, pp. 28-35, 2013.

[6] R. L. Gordon, "A technique for control of traffic at critical intersections," Transportation Science, vol. 3, no. 4, pp. 279-288, 1969.

[7] C. Oh and S. G. Ritchie, "Anonymous vehicle tracking for realtime traffic surveillance and performance on signalized arterials," Transportation Research Record Journal of the Transportation Research Board, vol. 1826, no. 1, pp. 37-44, 2001.

[8] J. Huang, G. Li, Q. Wang, and H. Yu, "Real time delay estimation for signalized intersection using transit vehicle positioning data," in International Conference on ITS Telecommunications, IEEE, Tampere, Finland, pp. 216-2212013.

[9] H. J. Cho, Y. J. Jou, and C.-L. Lan, “Time dependent origindestination estimation from traffic count without prior information," Networks \& Spatial Economics, vol. 9, no. 2, pp. 145-170, 2009.

[10] B. Gunay, "Using automatic number plate recognition technology to observe drivers' headway preferences," Journal of Advanced Transportation, vol. 46, no. 4, pp. 305-317, 2012.

[11] H. Hashemi and K. F. Abdelghany, "Real-time traffic network state estimation and prediction with decision support capabilities: application to integrated corridor management," Transportation Research Part C, vol. 73, pp. 128-146, 2016.

[12] X. Sun and Y. Miyanaga, "Dynamic time warping for speech recognition with training part to reduce the computation," International Symposium on Signals, vol. 18, no. 2, pp. 89-96, 2014.

[13] Y. L. Hsu, C. L. Chu, Y. J. Tsai, and Wang, "An inertial pen with dynamic time warping recognizer for handwriting and gesture recognition," Sensors Journal IEEE, vol. 15, no. 1, pp. 154-163, 2015.

[14] J. G. Proakis and D. K. Manolakis, Digital Signal Processing, Pearson, 4th edition, 2006.

[15] S. Mallat and Z. Zhang, "Matching pursuit with time-frequency dictionary," IEEE Transactions on Signal Processing, vol. 41, no. 12 , pp. 3397-3415, 1993.

[16] H. N. Yagoda, E. H. Principe, C. E. Vick, and B. Leonard, "Subdivision of signal systems into control areas," Traffic Engineering - Institute of Transportation Engineers, vol. 43, no. 12 , pp. 42-45, 1973.

[17] H. Abouaïssa, M. Fliess, and C. Join, "On ramp metering: towards a better understanding of ALINEA via model-free control," International Journal of Control, vol. 90, no. 5, pp. 1018-1026, 2017.

[18] T. W. Liao, "Clustering of time series data-a survey," Pattern Recognition, vol. 38, no. 11, pp. 1857-1874, 2005.

[19] T. T. Tin, N. T. Hien, and V. T. Vinh, "Measuring similarity between vehicle speed records using dynamic time warping," in Seventh International Conference on Knowledge \& Systems Engineering, IEEE, 2015.

[20] Z. Zhang, T. Zhao, X. Ao, and H. Yuan, "A vehicle speed estimation algorithm based on dynamic time warping approach," IEEE Sensors Journal, vol. 17, no. 8, pp. 2456-2463, 2017.

[21] F. Lu, K. Liu, Y. Duan, and S. Cheng, "Modelling the heterogeneous traffic correlations in urban road systems using traffic-enhanced community detection approach," Physica A Statistical Mechanics \& Its Applications, vol. 501, pp. 227-237, 2018.
[22] L. Yan, "Routes classification method at intersections group using wavelet transform and spectrum analysis," Journal of Southeast University, vol. 42, no. 1, pp. 168-172, 2012.

[23] J. Guo, Q. Xiong, and S. Chen, "Division approach of traffic signal control sub-area," in International Conference on Information Engineering \& Computer Science, IEEE, 2009.

[24] N. E. Huang, Z. Shen, S. R. Long et al., "The empirical mode decomposition and the Hilbert spectrum for nonlinear and non-stationary time series analysis," Proceedings Mathematical Physical \& Engineering Sciences, vol. 454, no. 1971, pp. 903-995, 1998.

[25] A. O. Boudraa and J. C. Cexus, "EMD-based signal filtering," IEEE Transactions on Instrumentation \& Measurement, vol. 56, no. 6, pp. 2196-2202, 2007.

[26] G. N. Stainhaouer and G. Carayannis, "New parallel implementations for DTW algorithms," Acoustics Speech \& Signal Processing IEEE Transactions, vol. 38, no. 4, pp. 705-711, 1990.

[27] E. Keogh and C. A. Ratanamahatana, "Exact indexing of dynamic time warping," Knowledge \& Information Systems, vol. 7, no. 3, pp. 358-386, 2005.

[28] L. Wei and E. Keogh, "Semi-supervised time series classification," in Proceedings of the 12th ACM SIGKDD International Conference on Knowledge Discovery and Data Mining, ACM, New York, NY, USA, 2006.

[29] D. J. Berndt and J. Clifford, "Using dynamic time warping to find patterns in time series," KDD Workshop, vol. 10, no. 16, 1994.

[30] J. A. Hartigan and M. A. Wong, "Algorithm AS 136: a k-means clustering algorithm," Applied Statistics, vol. 28, no. 1, pp. 100$108,1979$.

[31] K.-S. Chuang, H.-L. Tzeng, S. Chen, J. Wu, and T.-J. Chen, "Fuzzy c-means clustering with spatial information for image segmentation," Computerized Medical Imaging and Graphics, vol. 30, no. 1, pp. 9-15, 2006.

[32] R. Yu and M. Abdel-Aty, "An optimal variable speed limits system to ameliorate traffic safety risk," Transportation Research Part C: Emerging Technologies, vol. 46, pp. 235-246, 2014.

[33] Z. Adam, M. Abbas, and Y. Li, "Critical routes identification method using wavelet filtering," in International IEEE Conference on Intelligent Transportation Systems, IEEE, pp. 337-341, 2010. 


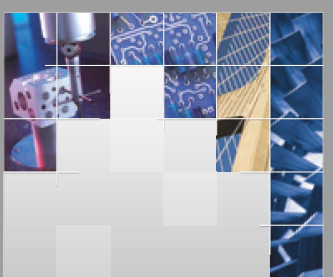

\section{Enfincering}
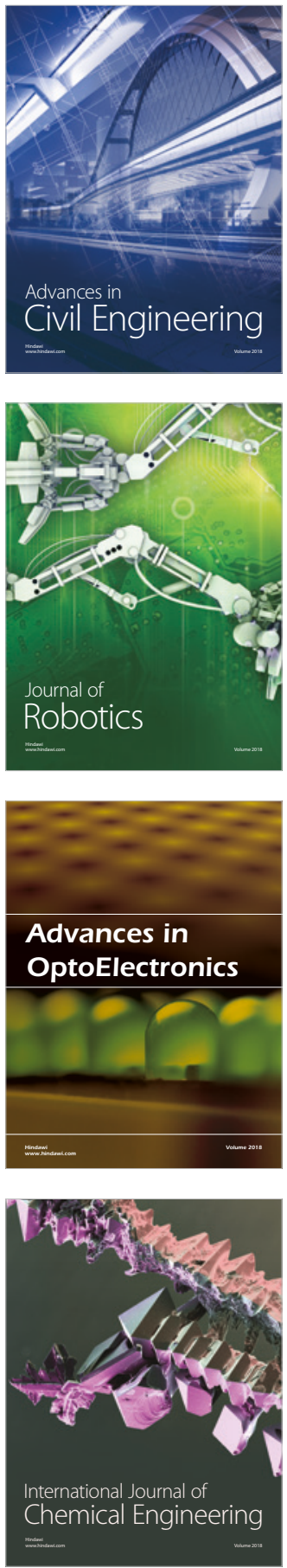

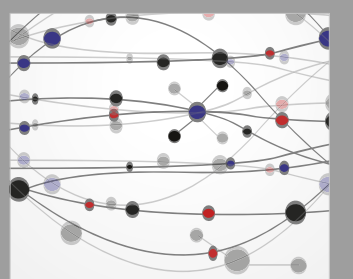

\section{Rotating \\ Machinery}

The Scientific World Journal

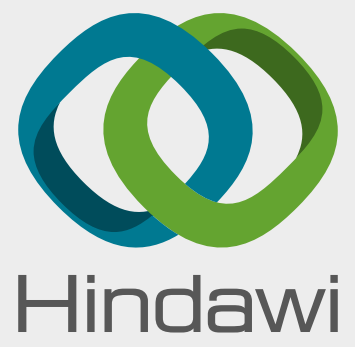

Submit your manuscripts at

www.hindawi.com
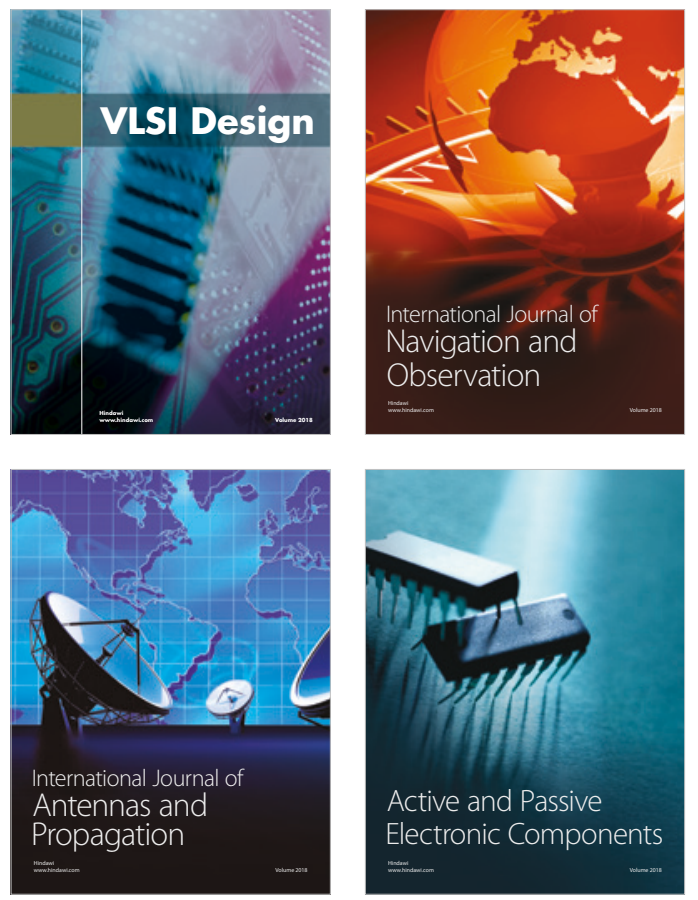
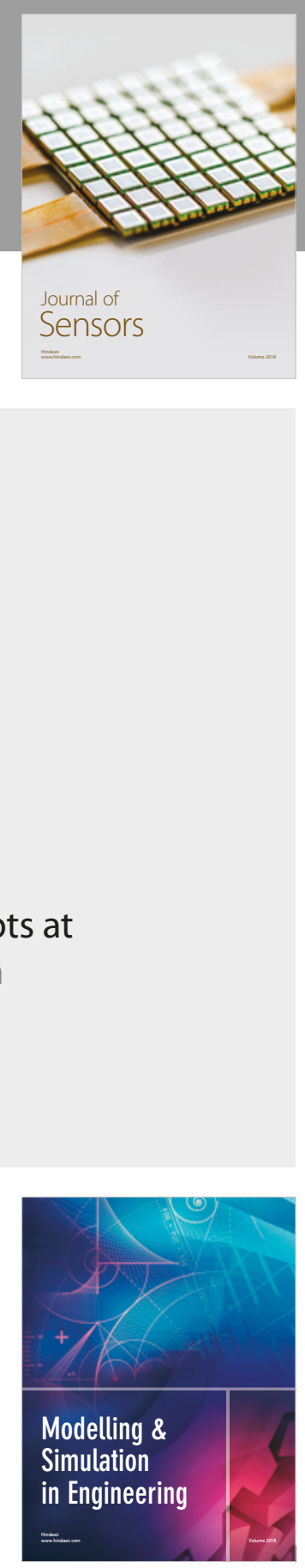

\section{Advances \\ Multimedia}
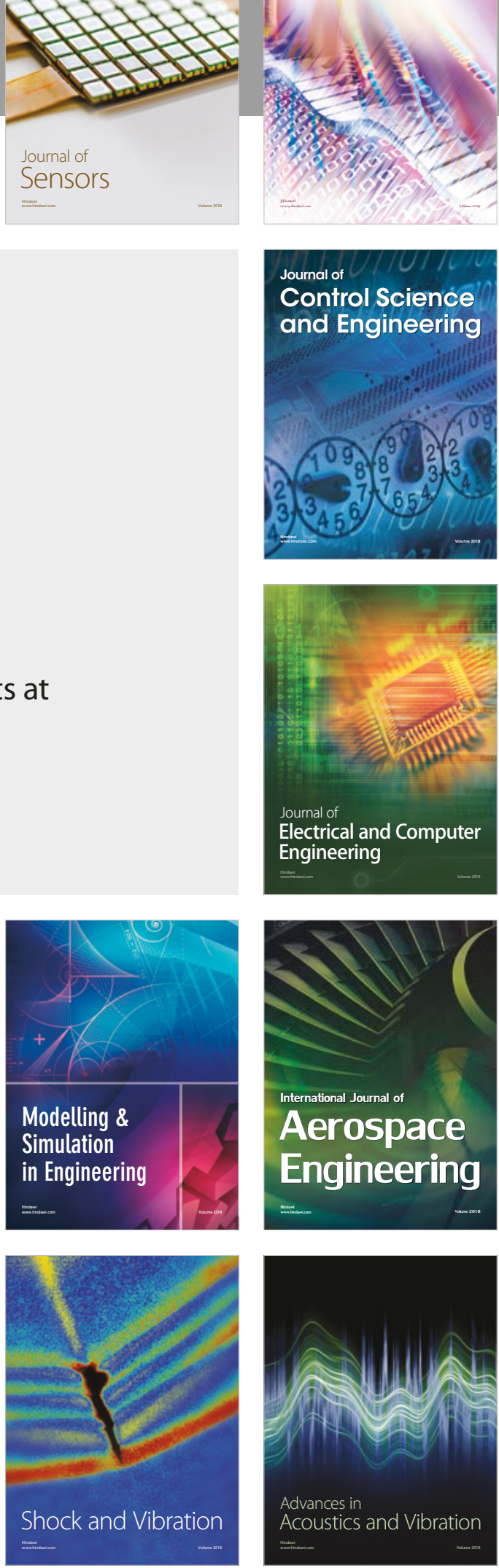